\title{
MOVES III. Simultaneous X-ray and ultraviolet observations unveiling the variable environment of the hot Jupiter HD 189733b
}

\author{
V. Bourrier, ${ }^{1 \star}$ P. J. Wheatley ${ }^{\oplus},{ }^{2,3 \star}$ A. Lecavelier des Etangs, ${ }^{4 \star}$ G. King, ${ }^{2,3}$ \\ T. Louden ${ }^{\odot},{ }^{2,3}$ D. Ehrenreich, ${ }^{1}$ R. Fares,${ }^{5}$ Ch. Helling, ${ }^{6,7}$ J. Llama, ${ }^{8}$ \\ M. M. Jardine ${ }^{\oplus 9}$ and A. A. Vidotto ${ }^{\oplus 10}$ \\ ${ }^{1}$ Observatoire de l'Université de Genève, Chemin des Maillettes 51, Versoix CH-1290, Switzerland \\ ${ }^{2}$ Department of Physics, University of Warwick, Gibbet Hill Road, Coventry CV4 7AL, UK \\ ${ }^{3}$ Centre for Exoplanets and Habitability, University of Warwick, Gibbet Hill Road, Coventry CV4 7AL, UK \\ ${ }^{4}$ Institut d'Astrophysique de Paris, CNRS, UMR 7095 and Sorbonne Universités, UPMC Paris 6, 98 bis bd Arago, F-75014 Paris, France \\ ${ }^{5}$ Physics Department, United Arab Emirates University, P.O. Box 15551 Al-Ain, United Arab Emirates \\ ${ }^{6}$ Centre for Exoplanet Science, School of Physics and Astronomy, University of St Andrews, North Haugh, St Andrews KY16 9SS, UK \\ ${ }^{7}$ SRON Netherlands Institute for Space Research, Sorbonnelaan 2, NL-3584 CA Utrecht, the Netherlands \\ ${ }^{8}$ Lowell Observatory, 1400 West Mars Hill Road, Flagstaff, AZ 86001, USA \\ ${ }^{9}$ SUPA, School of Physics and Astronomy, North Haugh, St Andrews, Fife KY16 9SS, UK \\ ${ }^{10}$ School of Physics, Trinity College Dublin, College Green, Dublin-2, Ireland
}

Accepted 2020 January 23. Received 2020 January 23; in original form 2019 September 17

\begin{abstract}
In this third paper of the MOVES (Multiwavelength Observations of an eVaporating Exoplanet and its Star) programme, we combine Hubble Space Telescope far-ultraviolet (FUV) observations with XMM-Newton/Swift X-ray observations to measure the emission of HD 189733 in various FUV lines, and its soft X-ray spectrum. Based on these measurements we characterize the interstellar medium towards HD 189733 and derive semisynthetic XUV spectra of the star, which are used to study the evolution of its high-energy emission at five different epochs. Two flares from HD 189733 are observed, but we propose that the long-term variations in its spectral energy distribution have the most important consequences for the environment of HD 189733b. Reduced coronal and wind activity could favour the formation of a dense population of $\mathrm{Si}^{2+}$ atoms in a bow-shock ahead of the planet, responsible for preand in-transit absorption measured in the first two epochs. In-transit absorption signatures are detected in the Lyman $\alpha$ line in the second, third, and fifth epochs, which could arise from the extended planetary thermosphere and a tail of stellar wind protons neutralized via chargeexchange with the planetary exosphere. We propose that increases in the X-ray irradiation of the planet, and decreases in its EUV irradiation causing lower photoionization rates of neutral hydrogen, favour the detection of these signatures by sustaining larger densities of $\mathrm{H}^{0}$ atoms in the upper atmosphere and boosting charge-exchanges with the stellar wind. Deeper and broader absorption signatures in the last epoch suggest that the planet entered a different evaporation regime, providing clues as to the link between stellar activity and the structure of the planetary environment.
\end{abstract}

Key words: planets and satellites: individual: HD189733b-planet-star interactions - ISM: clouds - stars: chromospheres, coronae-stars: individual: HD 189733 - techniques: spectroscopic. 


\section{INTRODUCTION}

Nearly half of the known exoplanets orbit within 0.1 au from their star. At such close distances, the nature and evolution of these planets is shaped by interactions with their host star (irradiation, tidal effects, and magnetic fields). In particular, the deposition of stellar X-ray and extreme ultraviolet radiation (XUV) into an exoplanet upper atmosphere can lead to its hydrodynamic expansion and substantial escape (e.g. Lammer et al. 2003; Vidal-Madjar et al. 2003; Lecavelier des Etangs et al. 2004; Yelle 2004; García Muñoz 2007; Koskinen et al. 2010; Johnstone et al. 2015). Atmospheric loss is considered as one of the main processes behind the deficit of Neptune-mass planets at close orbital distances (the so-called hot Neptune desert, e.g. Lecavelier des Etangs 2007; Davis \& Wheatley 2009; Szabó \& Kiss 2011; Lopez, Fortney \& Miller 2012; Beaugé \& Nesvorný 2013; Lopez \& Fortney 2013; Owen \& Wu 2013; Jin et al. 2014; Kurokawa \& Nakamoto 2014; Lundkvist et al. 2016). These planets are large enough to capture much of the stellar energy, but in contrast to hot Jupiters are not massive enough to retain their escaping atmospheres (e.g. Hubbard et al. 2007; Lecavelier des Etangs 2007; Ehrenreich, Lecavelier des Etangs \& Delfosse 2011). The missing hot Neptunes could have lost their entire atmosphere via evaporation, evolving into bare rocky cores at the lower radius side of the desert (e.g. Lecavelier des Etangs et al. 2004; Owen \& Jackson 2012). This scenario is strengthened by the recent observations of warm Neptunes at the border of the desert, on the verge of (Kulow et al. 2014; Bourrier, Ehrenreich \& Lecavelier des Etangs 2015; Ehrenreich et al. 2015; Bourrier et al. 2016; Lavie et al. 2017) or undergoing (Bourrier et al. 2018b) considerable massloss. Because they survive more extreme conditions than lower mass gaseous exoplanets, hot Jupiters are particularly interesting targets to study star-planet interactions. Their upper atmosphere can be substantially ionized because of stellar photoionization (e.g. Schneiter et al. 2016), which could help the formation of reconnections between the stellar and planetary magnetospheres that would enhance stellar activity (e.g. Cuntz, Saar \& Musielak 2000; Shkolnik, Walker \& Bohlender 2003; Ip, Kopp \& Hu 2004; Shkolnik et al. 2008; although see Poppenhaeger \& Schmitt 2011; Scandariato et al. 2013; Llama \& Shkolnik 2015 for the difficulties to detect such signatures). Atmospheric escape of neutral hydrogen and metal species has been detected via transmission spectroscopy for several Jupiter-mass planets, bringing information about their upper atmosphere and the stellar environment (HD 209458b, VidalMadjar et al. 2003, 2004, 2008; Ehrenreich et al. 2008; Ben-Jaffel \& Sona Hosseini 2010; Linsky et al. 2010; Schlawin et al. 2010; VidalMadjar et al. 2013; Ballester \& Ben-Jaffel 2015; HD 189733b, Lecavelier des Etangs et al. 2010, 2012; Bourrier et al. 2013; $55 \mathrm{Cnc}$ b, Ehrenreich et al. 2012; WASP-12b, Fossati et al. 2010; Haswell et al. 2012). Shocks could for example form ahead of hot Jupiters because of the interaction between the stellar wind and the planetary outflow or magnetosphere (Vidotto, Jardine \& Helling 2010; Cohen et al. 2011; Llama et al. 2013; Tremblin \& Chiang 2013; Matsakos, Uribe \& Königl 2015)

The HD 189733 system offers the possibility to study these various interactions (Table 1). It is a binary system with a $\mathrm{K} 2 \mathrm{~V}$ dwarf (HD 189733 A, hereafter HD 189733) and a M4V dwarf (HD 189733 B; Bakos et al. 2006) at a mean separation of $\sim 220$ au. The bright primary $(V=7.7)$ hosts a transiting hot Jupiter at 0.03 au (Bouchy et al. 2005), whose strong irradiation and large occultation area make it particularly favourable for atmospheric characterization (see Pino et al. 2018 and references inside for observations of the lower atmospheric layers). Recent transit observations in the near-infrared (Salz et al. 2018) revealed absorption by helium in an extended but compact thermosphere. Transit observations in the far-ultraviolet (FUV) previously revealed absorption by a dense and hot layer of neutral oxygen at higher altitudes (BenJaffel \& Ballester 2013). The close distance of HD 189733 to the Sun (19.8 pc) enables observations of the stellar Lyman $\alpha$ line with the Hubble Space Telescope (HST). Atmospheric escape of neutral hydrogen was first detected in the unresolved line with the HST Advanced Camera for Surveys (ACS) in two out of three epochs (Lecavelier des Etangs et al. 2010), and in the line resolved with the HST Space Telescope Imaging Spectrograph (STIS) in one out of two epochs (Lecavelier des Etangs et al. 2012; Bourrier et al. 2013). These observations provided the first indication of temporal variations in the physical conditions of an evaporating planetary atmosphere. Bourrier \& Lecavelier des Etangs (2013) attributed the high-velocity, blueshifted absorption signature detected by Lecavelier des Etangs et al. (2012) to intense charge-exchange between the planet exosphere and the stellar wind, proposing that an X-ray flare observed before the transit increased the atmospheric mass-loss and/or increased the density of the stellar wind. The latter scenario is favoured by thermal escape simulations from Chadney et al. (2017), who found that the energy input from a flare would not increase sufficiently the mass-loss. Interestingly the tentative detection of absorption by ionized carbon (Ben-Jaffel \& Ballester 2013) and excited hydrogen (Jensen et al. 2012; Cauley et al. 2015, 2016; Cauley, Redfield \& Jensen 2017; Kohl et al. 2018) before and during the transit of HD 189733b could be explained by the interaction of the stellar wind with the planetary magnetosphere or escaping material. The observed temporal variability in the atmospheric escape of neutral and excited hydrogen is likely linked to the high-level of activity from the host star, which results in a fast-changing radiation, particle, and magnetic environment for the planet. Enhanced activity in the stellar chromosphere and transition region have also been observed after the planetary eclipse, and attributed to signatures of magnetic star-planet interactions (Pillitteri et al. 2010, 2011, 2014, 2015). Evidence for modulation in the CaII lines at the orbital period of the planet, during an epoch of strong stellar magnetic field, further supports this scenario (Cauley et al. 2018). The detectability of star-planet interactions in the HD 189733 system however remain uncertain, and intrinsic stellar variability and inadequate sampling has been proposed to explain the observed variations (Route 2019). These results show the need to study contemporaneously and in different epochs the upper atmosphere of HD 189733b and its high-energy environment.

In this context, we started a multiwavelength observational campaign of this system, in the frame of the MOVES collaboration (Multiwavelength Observations of an eVaporating Exoplanet and its Star, PI V. Bourrier). Observations of the star and the planet were obtained at similar epochs with ground-based and spaceborne instruments: in X-rays with the X-ray Multi-Mirror Mission (XMM-Newton) and Neil Gehrels Swift Observatory (Swift); in the UV with HST and XMM-Newton; in optical spectropolarimetry with NARVAL (Aurière 2003) and the Echelle SpectroPolarimetric Device for the Observation of Stars (ESPaDOnS; Donati 2003; Donati et al. 2006); and in radio with the Low-Frequency Array (LOFAR; van Haarlem et al. 2013). In MOVES I (Fares et al. 2017), we used optical spectropolarimetry of HD 189733 to reconstruct its surface and large-scale magnetic field in five epochs (2013 July, 2013 August, 2013 September, 2014 September, and 2015 June) We combined these results with data from Moutou et al. (2007), Fares et al. (2010) to study the evolution of the field over $9 \mathrm{yr}$, 
Table 1. Properties of the HD 189733 system fixed in our study.

\begin{tabular}{lccc}
\hline Parameters & Symbol & Value & Reference \\
\hline Distance from Earth & $D_{*}$ & $19.78 \pm 0.01 \mathrm{pc}$ & Gaia Collaboration (2018) \\
Star radius & $R_{*}$ & $0.780_{-0.024}^{+0.017} \mathrm{R}_{\odot}$ & Gaia Collaboration (2018) \\
Star mass & $M_{*}$ & $0.823 \pm 0.029 \mathrm{M}_{\odot}$ & Triaud et al. (2009) \\
Heliocentric stellar radial velocity & $\gamma_{* / \odot}$ & $-2.55 \pm 0.16 \mathrm{~km} \mathrm{~s}^{-1}$ & Gaia Collaboration (2018) \\
Planet-to-star radius ratio & $R_{\mathrm{p}} / R_{*}$ & $0.1571 \pm 0.0004$ & Baluev (2015) \\
Orbital period & $P_{\mathrm{p}}$ & $2.218575200 \pm 7.7 \times 10^{-8} \mathrm{~d}$ & Baluev (2015) \\
Transit centre & $T_{0}$ & $2453955.5255511 \pm 8.8 \times 10^{-6} \mathrm{BJD}_{\mathrm{TDB}}$ & Baluev (2015) \\
Scaled semimajor axis & $a_{\mathrm{p}} / R_{*}$ & $8.863 \pm 0.020$ & Agol et al. (2010) \\
Eccentricity & $e$ & 0 & Bouchy et al. (2005) \\
Argument of periastron & $\omega$ & $90^{\circ}$ & Bouchy et al. (2005) \\
Inclination & $i_{\mathrm{p}}$ & $85.710 \pm 0.024^{\circ}$ & Agol et al. (2010) \\
Impact parameter & $b$ & $0.6636 \pm 0.0019$ & Baluev (2015) \\
\hline
\end{tabular}

and found that its strength changed significantly even though its overall structure remained stable (toroidally dominated and with the same polarity). We showed that the magnetic environment is not homogeneous over the orbit of HD 189733b and varies between observing epochs. The resulting inhomogeneities of the stellar wind at the location of the planet (Llama et al. 2013) and its variations with the overall stellar magnetic field could cause variations in the structure of the planetary upper atmosphere and its UV transit light curve, on short time-scales (on the order of an orbital period) as well as longer time-scale (on the order of a year). In MOVES II (Kavanagh et al. 2019), we thus studied the stellar wind of HD 189733 and modelled the local particle and magnetic environment surrounding the planet. Our aim was to predict the radio environment of the system (emissions from the stellar wind and planet). From mid-2013 until mid-2015, we showed that, the yearly variation of the stellar wind, together with their inhomogeneities along the planet's orbit, indeed leads to significant variabilities in surrounding medium of HD 189733b. These results indicate that the best approach to characterize the upper atmosphere of a close-in planet, and to fully understand the physical interactions taking place with its star/stellar wind is to obtain, as much as possible, contemporaneous, multiwavelength observations of the system. In this paper (MOVES III), we perform a consistent analysis of five transit observations of HD 189733b in the FUV. We interpret these FUV observations together with X-ray observations of HD 189733 that were obtained simultaneously for all visits but the first. We present the observations and their analysis in Section 2. The search for variations in the $\operatorname{Lyman} \alpha$ line and other FUV stellar lines is described in Section 3. We derive and discuss the temporal evolution of the intrinsic Lyman $\alpha$ line and XUV spectrum of the star in Section 4. Our results are interpreted in terms of stellar evolution, planetary atmospheric escape, and starplanet interactions (SPI) in Section 5. Conclusions and perspectives are presented in Section 7.

\section{OBSERVATIONS AND DATA ANALYSIS}

\subsection{HST STIS observations}

We analysed transit observations of HD 189733b obtained in five independent epochs with the HST/STIS (Woodgate et al. 1998). Two archival data sets published in Lecavelier des Etangs et al. (2012), Bourrier et al. (2013) (Visits A and B) are combined with our three original data sets (PI: P.J. Wheatley; Visits C, D, and E). All data were obtained with the STIS/G140M grating (spectral range 1195 to $1248 \AA$, spectral resolution $\sim 20 \mathrm{~km} \mathrm{~s}^{-1}$ ), with the main purpose of searching for the transit of HD 189733b in the stellar Lyman $\alpha$ line. The log of the observations is given in Table 2 . Visits A, B, and C each consist of four consecutive HST orbits obtained before, during, and after the planetary transit. Visits D and E each consist of three consecutive HST orbits obtained before and during the transit. Data obtained in time-tagged mode were reduced with the CALSTIS pipeline (version 3.4, Hodge et al. 1998), which includes the flux and wavelength calibration, and divided in sub-

Table 2. Log of HD 189733 HST, XMM-Newton, and Swift observations.

\begin{tabular}{|c|c|c|c|c|c|c|c|c|}
\hline & \multirow[t]{2}{*}{ Date } & \multirow[t]{2}{*}{ Telescope } & \multicolumn{2}{|c|}{ Time from mid-transit (UT) } & \multicolumn{2}{|c|}{ Time $\left(\mathrm{BJD}_{\mathrm{TDB}}-2450000\right)$} & \multirow{2}{*}{$\begin{array}{c}\text { Number of } \\
\text { sub-exposures }\end{array}$} & \multirow{2}{*}{$\begin{array}{c}\text { Duration of } \\
\text { sub-exposures (s) }\end{array}$} \\
\hline & & & Start & End & Start & End & & \\
\hline Visit A & 2010-04-06 & $H S T$ & $-03: 17: 45$ & 02:00:53 & 5293.18907 & 5293.41035 & $6-7-7-7$ & $316-322-322-322$ \\
\hline Visit C & 2013-05-09/10 & $\begin{array}{c}H S T \\
X M M-\text { Newton }\end{array}$ & $\begin{array}{l}-02: 30: 37 \\
-05: 32: 22\end{array}$ & $\begin{array}{l}02: 39: 28 \\
05: 06: 48\end{array}$ & $\begin{array}{l}6422.47658 \\
6422.35036\end{array}$ & $\begin{array}{l}6422.69191 \\
6422.79422\end{array}$ & $\begin{array}{c}5-10-10-10 \\
41\end{array}$ & $\begin{array}{c}277-293-293-293 \\
970\end{array}$ \\
\hline Visit E & 2013-11-21 & $\begin{array}{c}H S T \\
X M M-\text { Newton }\end{array}$ & $\begin{array}{l}-02: 59: 01 \\
-06: 09: 10\end{array}$ & $\begin{array}{l}00: 41: 42 \\
05: 00: 10\end{array}$ & $\begin{array}{l}6617.69148 \\
6617.55943\end{array}$ & $\begin{array}{l}6617.84475 \\
6618.02424\end{array}$ & $\begin{array}{c}6-10-10 \\
43\end{array}$ & $\begin{array}{c}314-299-299 \\
970\end{array}$ \\
\hline
\end{tabular}

Note. The number and duration of sub-exposures are given for each HST orbit in STIS data. For Swift, sub-exposure correspond to individual observations taken on different spacecraft orbits. For XMM-Newton, individual exposures are very short and the values indicated correspond to our choice of binning. No X-ray data were obtained in Visit A. 

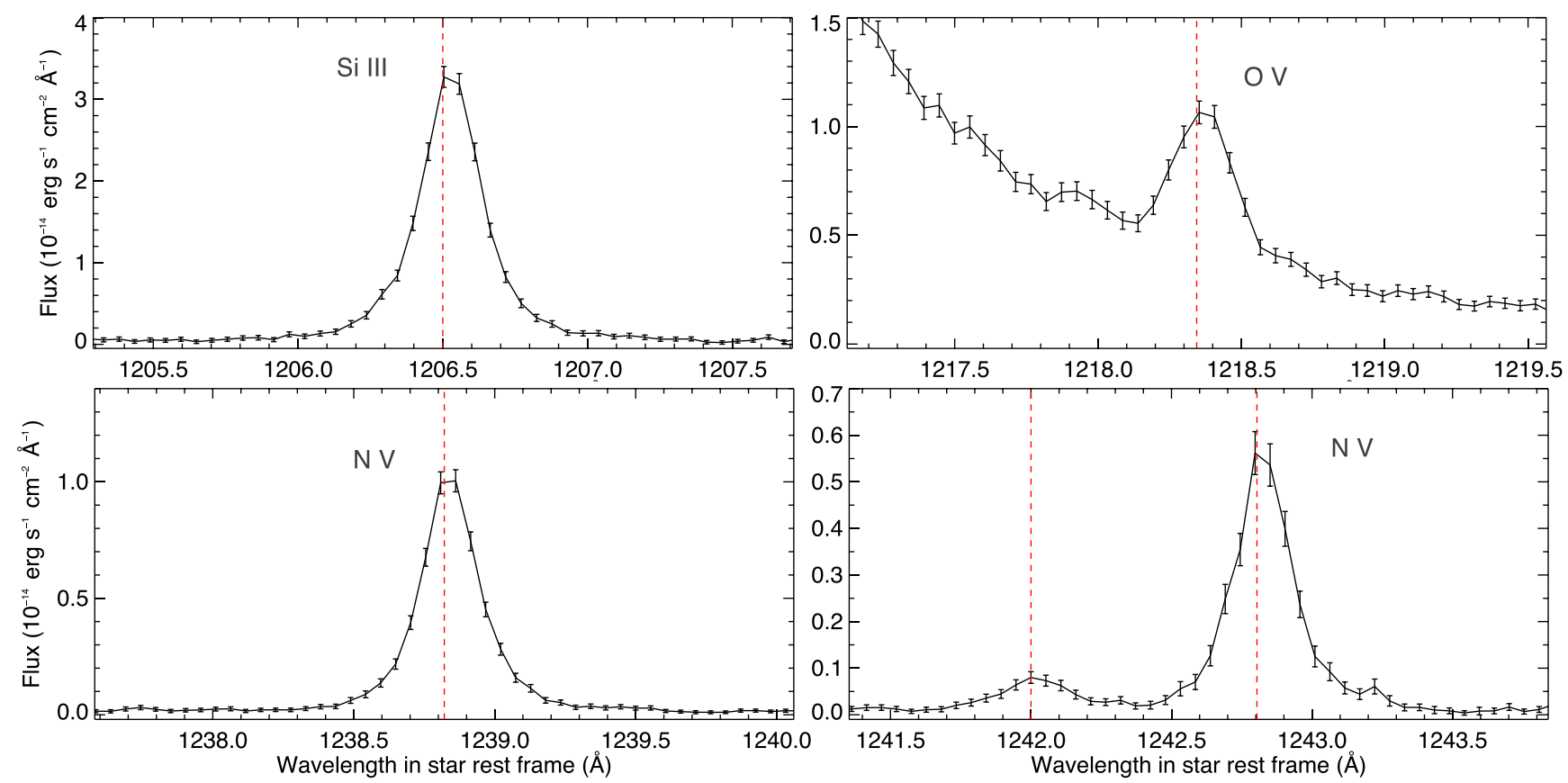

Figure 1. Spectral profiles of HD 189733 secondary FUV lines, averaged over the quiescent spectra in all visits. The vertical dashed red lines show the wavelengths of the transitions associated to each stellar line in the star rest frame.

exposures in each HST orbit. The first orbit (orbit 1) in each visit has a shorter scientific exposure because of target acquisition. In Visits A and B, acquisition-peakup exposures were performed at the start of orbits 2-3-4 in case the target needed recentring within the slit. This operation was no longer performed in Visits $\mathrm{C}$ to E, which further had the wavelength calibration exposures carried out during Earth occultation. This resulted in substantially longer scientific exposures in orbits 2-3-4 for those visits. The number of sub-exposures in the orbits of each visit was thus adjusted to keep a duration of $\sim 300 \mathrm{~s}$.

A K-type star like HD 189733 has no measurable continuum emission in the G140M spectral range. As can be seen in Fig. 1, we identified in each visit the following stellar emission lines: Lyman $\alpha$ line (1215.67 ̊), Si III (1206.5 ̊), O v (1218.3 ̊), Fe XII (1242.0 $\AA$ ), and the N V doublet (1242.8 and 1238.8 $\AA$ ). The stellar Lyman $\alpha$ line in the raw data is contaminated by geocoronal airglow emission from the upper atmosphere of Earth (Vidal-Madjar et al. 2003). CALSTIS corrects the final 1D spectra for airglow contamination, but it is recommended to treat with caution the regions where the airglow is stronger than the stellar flux (e.g. Bourrier et al. 2017, 2018b). The strength and position of the airglow varies with the epoch of observation, and after preliminary analyses of the Lyman $\alpha$ spectra we identified the wavelength windows shown in Fig. 2 as unreliable. Airglow is low enough in Visit B that the full stellar line profile could be analysed.

\subsection{HST STIS calibrations}

The HST experiences thermal variations over its orbit, which induce variations in the telescope throughput and modify the balance of the flux measured with STIS in each orbit (e.g. Brown et al. 2001; Sing et al. 2008; Huitson et al. 2012). This 'breathing' effect is detected in all visits (Fig. 3). As in previous measurements with the G140M grating (e.g. Bourrier et al. 2013; Ehrenreich et al. 2015; Bourrier et al. 2017), the shape and amplitude of the breathing variations change between visits but the orbit-to-orbit variations within a single visit are both stable and highly repeatable, allowing for an efficient correction. Particular care must be taken, however, with the first orbit. Various operations (Section 2.1) make its scientific exposure shorter and shifted to later HST orbital phases compared to subsequent exposures (Fig. 3). As a result, the flux unbalanced by breathing variations has a different average over the first orbit compared to later orbits. By accounting for this bias we improve on the correction performed for Visits A and B by Lecavelier des Etangs et al. (2012) and Bourrier et al. (2013), who assumed the breathing did not change the average flux over each orbit.

We fitted a breathing model based on Bourrier et al. (2017) to the sub-exposure spectra integrated over the entire Lyman $\alpha$ line (1214.0-1217.3 $\AA$ minus the range contaminated by the airglow). This choice is motivated by the achromaticity of the breathing variations, and by the need for a high signal-to-noise ratio (SNR) to ensure an accurate correction. The breathing was modelled as a polynomial function phased with the period of the HST around the Earth $\left(P_{\mathrm{HST}}=96 \mathrm{~min}\right)$. The nominal flux unaffected by the breathing effect was allowed to vary for each HST orbit, to prevent the overcorrection of putative orbit-to-orbit variations caused by the star or the planet. We none-the-less excluded from the fit sharp flux variations caused by a flare at the end of orbit 2 in Visit C (Section 3.3). The breathing model was oversampled in time and averaged within the time window of each sub-exposure before comparison with the data. We used the Bayesian Information Criterion (BIC; Liddle 2007) as a merit function to determine the best polynomial degree for the breathing variations. The best-fitting models, shown in Fig. 3, were obtained for degrees of 1, 2, 3, 1, and 4 in visits A to E, respectively. Spectra in each sub-exposure were corrected by the value of the best-fitting breathing function at the time of mid-exposure.

After correcting the wavelength tables of the spectra for the heliocentric radial velocity of HD 189733 (Table 1), we found 

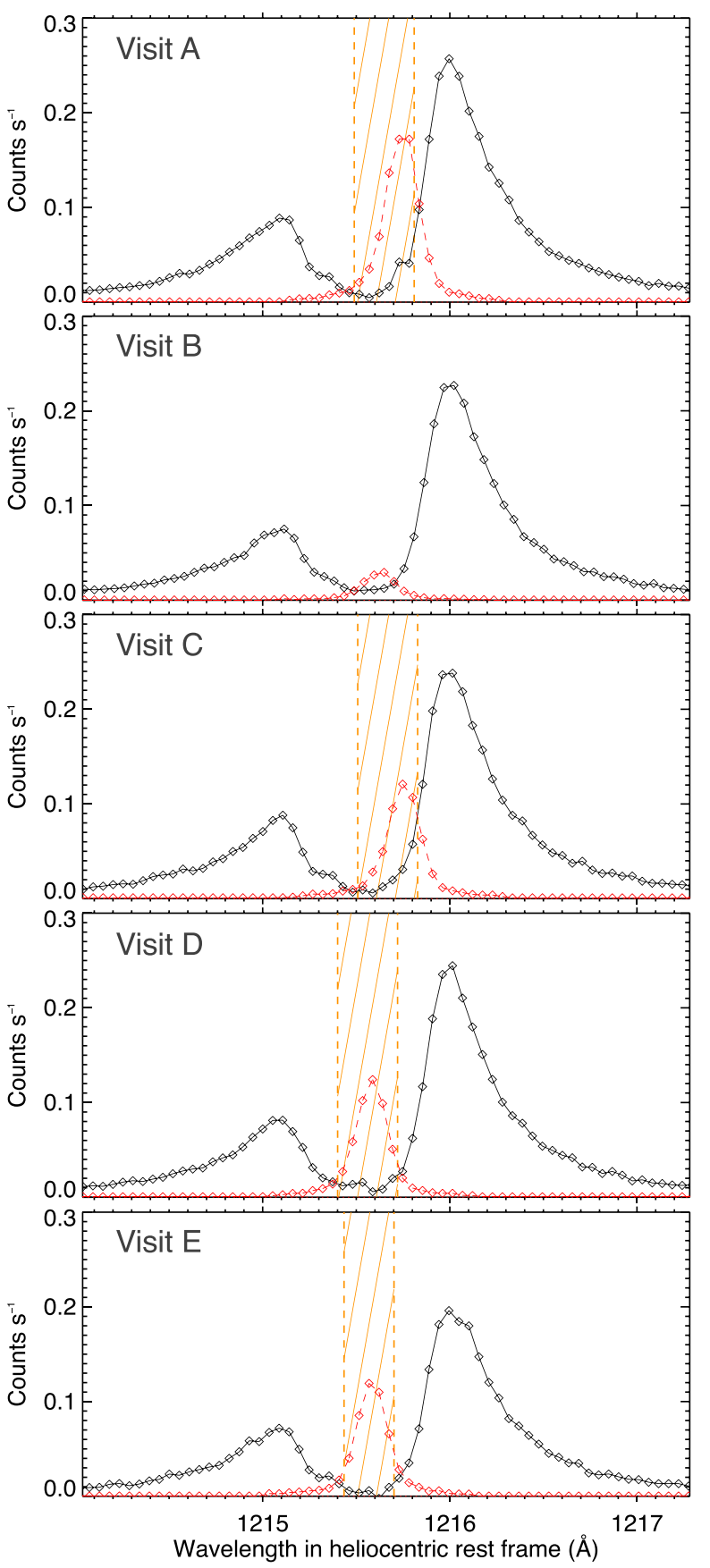

Figure 2. Raw spectra of HD 189733 Lyman $\alpha$ line (black profiles) after correction for the geocoronal emission line (red profiles). Spectra are averaged over all orbits in each visit. The dashed orange regions show wavelengths window excluded from our analyses because of strong airglow contamination.

that some of the stellar lines were redshifted with respect to their expected rest wavelength relative to the star, as previously noted by Bourrier et al. (2013). Contrary to these authors we concluded that this redshift has a stellar rather than instrumental origin (see Section 4), which is why the velocity ranges we report hereafter (defined in the star rest frame without further correction) are slightly different than in Lecavelier des Etangs et al. (2012) and Bourrier et al. (2013).
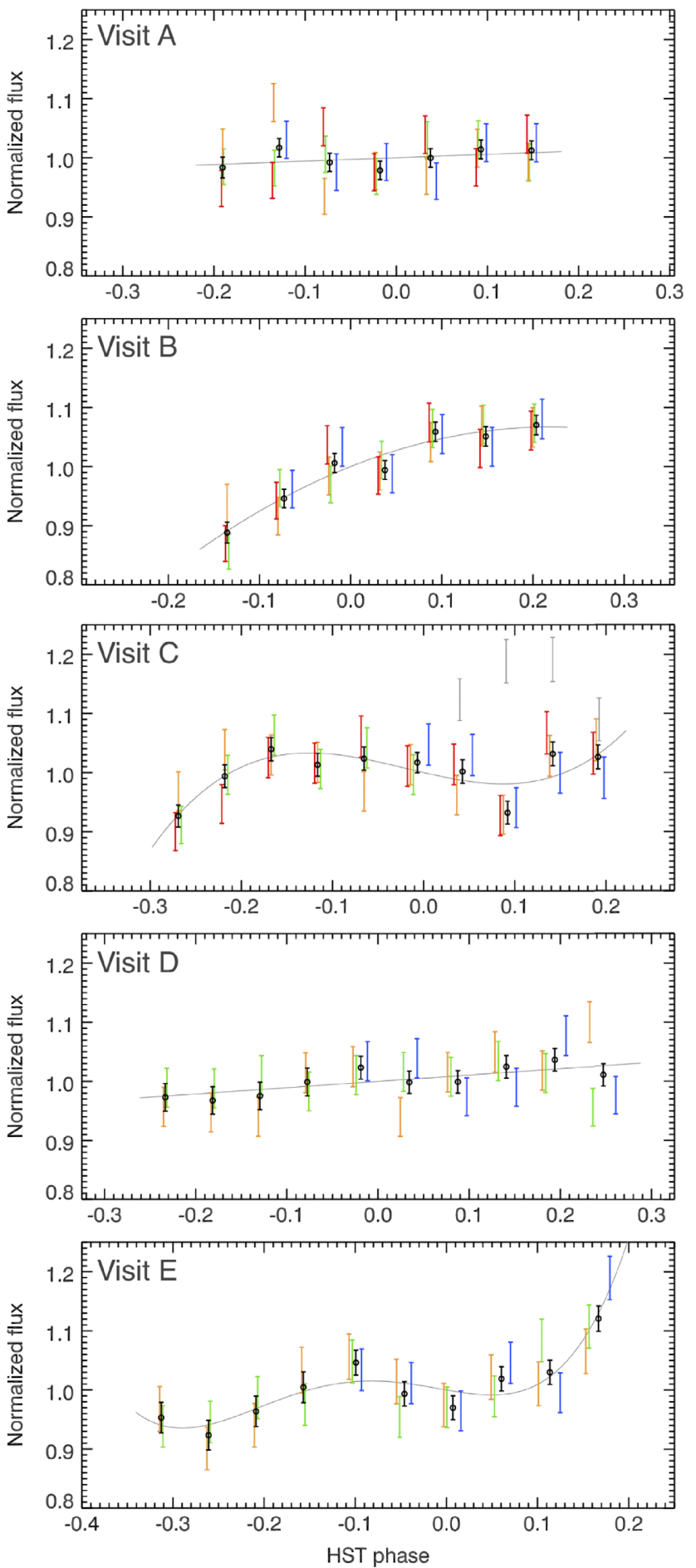

Figure 3. Lyman $\alpha$ fluxes for sub-exposures integrated over the entire line and phase-folded on the HST orbital period $\left(P_{\mathrm{HST}}=96 \mathrm{~min}\right)$. Phase is between -0.5 and 0.5 . The solid grey lines correspond to the best-fitting breathing model to the data, which have been scaled to the same nominal flux for the sake of comparison. The black points show sub-exposures binned manually to highlight the breathing trend. The colours correspond to HST consecutive orbits in each visit (orbits 1 to 4 are plotted in blue, green, orange, red). The four grey sub-exposures in Visit $\mathrm{C}$ are affected by a flare and have been excluded from the fit. 

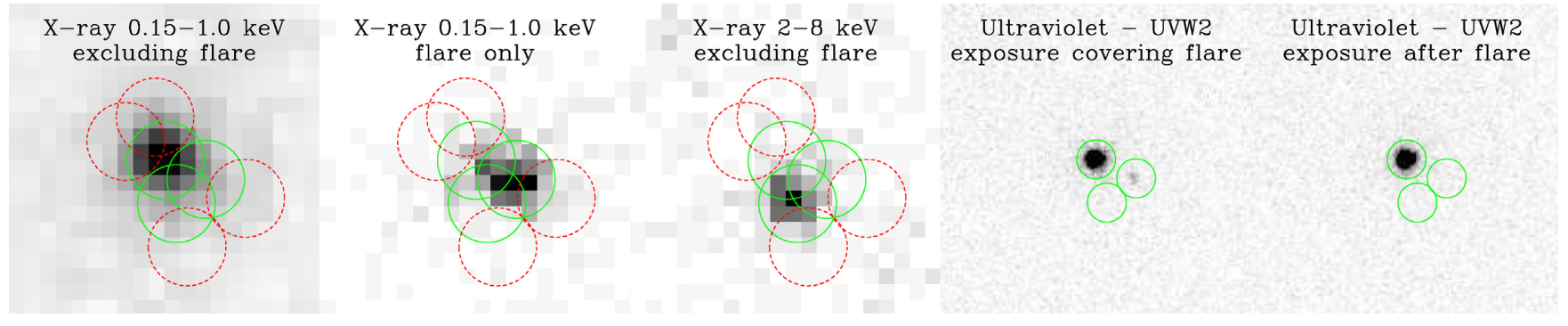

Figure 4. Plot showing the extraction regions for assessing contributions from the three X-ray sources (HD 189733, top left; HD 189733B, right; background $\mathrm{X}$-ray source, bottom left) overplotted on various images from the XMM-Newton visit D observations. The green circles show the source extraction regions, and red circles the regions used to estimate the contamination of each source by the two others (see the text). The first three plots show EPIC-pn data, the last two plots show $O M$ data.

\subsection{XMM-Newton and Swift observations}

We analysed spectra and light curves from three observations of HD 189733 taken with the EPIC-pn camera (Strüder et al. 2001) onboard XMM-Newton in 2013 (ObsID: 0692290201, 0692290301, 0692290401; PI: Wheatley), contemporaneous with HST visits C, $\mathrm{D}$, and E. We also looked at data from the Optical Monitor, but for the sole purpose of flare identification, a full analysis will be presented in an independent paper. The log of the observations is given in Table 2. The observations were made with the thin optical blocking filter in order to maximize the response to soft X-rays, and in small window mode in order to avoid pile-up. The source was very strongly detected in all three observations. The data were reduced in the standard way using the Scientific Analysis System (SAS 16.0.0).

There are two other X-ray sources near to HD 189733 on the sky, as identified with Chandra (Poppenhaeger, Schmitt \& Wolk 2013): the companion M dwarf HD 189733B, and a background source. The three form a roughly equilateral triangle on the sky, with angular separations of about 12 arcsec. Fig. 4 shows how the contamination of each of the sources by the others was considered. Using small source extraction regions of 10 arcsec radius (green circles in Fig. 4), we subtracted equivalently sized regions from the opposite side of the contaminating sources (red dashed circles to estimate the count rate of each component separately. This analysis showed that the contribution of HD 189733B is negligible at all energies except during a single flaring period in Visit D, which we excluded from our analyses (see Section 3). HD 189733 and the background source are spatially resolved in Chandra observations published by Poppenhaeger et al. (2013). The comparison of their spectra show that the contribution of the background source is negligible at energies below about $1.2 \mathrm{keV}$, where HD 189733 emits most of its X-ray energy (Section 4.3). Therefore we used the larger 15 arcsec regions to extract the total count rate, and excluded energies above $1.2 \mathrm{keV}$ to characterize the $\mathrm{X}$-ray emission of HD 189733 (the EPIC-pn camera observes from 0.16 to $15 \mathrm{keV}$ ). We note that most of the X-ray flux is emitted at the softer energies within the $0.166-1.2 \mathrm{keV}$ energy range (see spectrum in Fig. 18), and no significant differences are observed in the variations of the integrated X-ray flux over time when including or excluding harder energies.

We also analysed a set of observations of HD 189733 taken with the XRT instrument (Burrows et al. 2005) on Swift (ObsID: 00036406010 to 00036406017 ; PI: Wheatley), simultaneous with $H S T$ visit B. These observations were previously presented by Lecavelier des Etangs et al. (2012), who identified an X-ray flare about $8 \mathrm{~h}$ before the primary transit of the planet. The XRT instrument observes from 0.2 to $10 \mathrm{keV}$. As with the XMM-Newton data the background source could contaminate the flux at high energies, which were excluded from our analysis of HD189733. For these data we used source and background regions of radius 30 and 100 arcsec, respectively. These were extracted using the XSELECT program. ${ }^{1}$

\section{SEARCH FOR FUV SPECTRAL LINE VARIATIONS}

We searched for flux variations in the lines of HD 189733, which would arise from absorption by the planetary atmosphere or from stellar activity. Spectra were first compared two by two in each visit to identify those showing no significant variations, which could be considered as representative of the quiescent, unocculted stellar lines. This was done by searching for all features characterized by flux variations with $\mathrm{S} / \mathrm{N}$ larger than 3 , and extending over more than 3 pixels ( $\sim 0.16 \AA$, larger than STIS/G140M spectral resolution). For the brightest lines (Lyman $\alpha$, Si III, and the co-added lines of the $\mathrm{N} \mathrm{V}$ doublet), we compared spectra averaged not only over each orbit but also over groups of sub-exposures. Once stable spectra were identified for each line, they were coadded into a master quiescent spectrum for each visit, which was used to characterize the features detected in the variable spectra more precisely. We present the results of these analyses in the following sections, along with the $\mathrm{X}$-ray light curves measured in Visits B-E to help disentangling the stellar and planetary variations. The Swift light curve (visit B) covers the energy range 0.3 to $1.2 \mathrm{keV}$, and the $X M M-N e w t o n$ light curves (visits $\mathrm{C}$ to $\mathrm{E}$ ) the energy range 0.16 to $1.2 \mathrm{keV}$.

The $\mathrm{OV}$ line was analysed after correcting the spectra for the red wing of HD 189733 Lyman $\alpha$ line using a polynomial model specific to each visit (Fig. 1). However the corrected $\mathrm{OV}$ line is too faint to be analysed spectrally, and we found no significant variations in the flux integrated over the entire line in any of the visits. Therefore we do not discuss variations in the $\mathrm{O} v$ hereafter.

\subsection{Visit A - 2010 April 6}

The Si III line shows a similar profile in orbits 1 and 4, which is different from the similar profile it shows in orbits 2 and 3 (Fig. 5). We consider that the first group is representative of the intrinsic stellar line because its out-of-transit exposures show a symmetrical line profile. In contrast, mirroring the line in orbits 2 and 3 reveals that it is distorted and misses flux in its peak and red wing. Compared

\footnotetext{
${ }^{1}$ https://heasarc.gsfc.nasa.gov/ftools/xselect/
} 

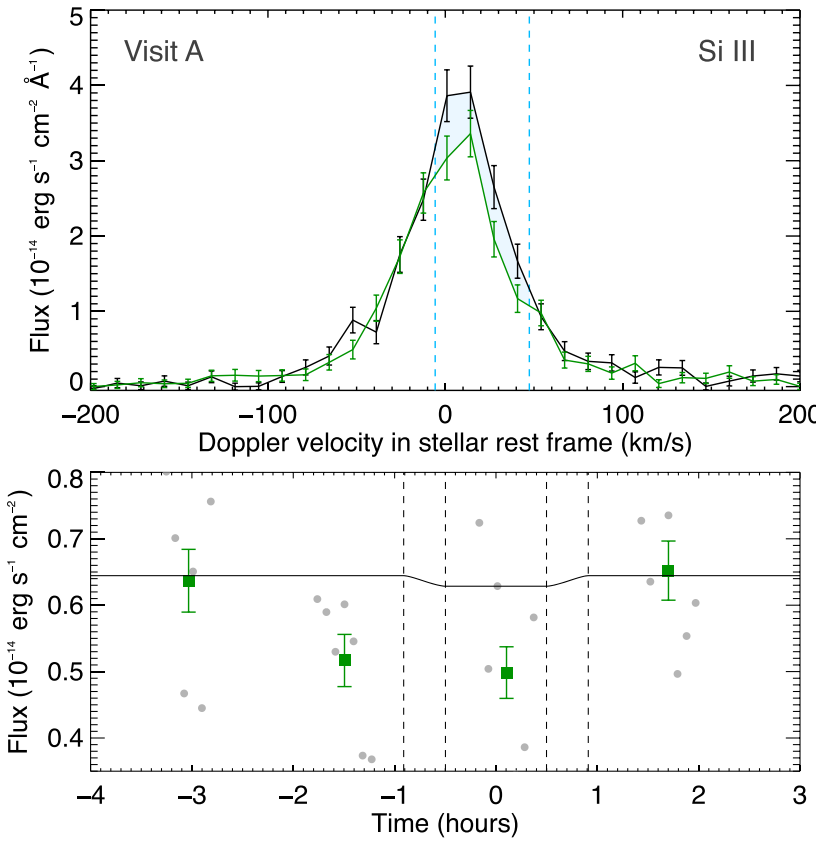

Figure 5. Si III line variations in Visit A. Top panel: Stellar line profile, averaged over orbits 1 and 4 (black) and over orbits 2 and 3 (green). The spectral range showing absorption is highlighted in blue. Bottom panel: Flux integrated over the absorbed spectral range, as a function of time relative to the planet transit. The green squares correspond to entire orbits, while grey discs stand for sub-exposures. The solid black line is the optical planetary light curve, with contacts shown as dashed vertical lines.

to orbits $1+4$ this corresponds to an absorption of $21.2 \pm 5.8$ per cent within -5.7 to $47.4 \mathrm{~km} \mathrm{~s}^{-1}$, which occurs in exposures obtained just before and during the planetary transit (Fig. 5). This localized absorption signature was not detected by Bourrier et al. (2013), who grouped pre-transit observations and focused on variations over the entire Si III line. Other parts of the Si III line remain stable during the visit.

We do not detect any significant variations in the $\mathrm{NV}$ lines, although we note that their wings are marginally brighter in the first orbit compared to subsequent exposures.

We also do not detect any significant variations in the Lyman $\alpha$ line, in agreement with Lecavelier des Etangs et al. (2012), Bourrier et al. (2013), and Guo \& Ben-Jaffel (2016). We show in Fig. 6 the comparison between the master out-of-transit spectrum and that obtained during the optical transit, when absorption by an extended exosphere of neutral hydrogen is expected to be strongest (Bourrier \& Lecavelier des Etangs 2013).

\subsection{Visit B - 2011 September 7/8}

The Si III line shows a similar profile in orbits 1 and 2, which is different from the similar profile it shows in orbits 3 and 4 (Fig. 7). We consider the second group to be representative of the stellar Si III line, because its profile is nearly identical to that of the quiescent line in Visit A. In contrast the core of the line shows significant absorption in orbits $1+2(28.3 \pm 5.5$ percent within -26.8 to $26.3 \mathrm{~km} \mathrm{~s}^{-1}$ ), which might extend further in the red wing. These variations were reported by Bourrier et al. (2013), and occur in the two orbits before the planetary transit (Fig. 7).

No variations were detected in the brightest line of the $\mathrm{N} v$ doublet ( $\lambda 1239)$. The N V $\lambda 1243$ line, however, shows a lower flux in the
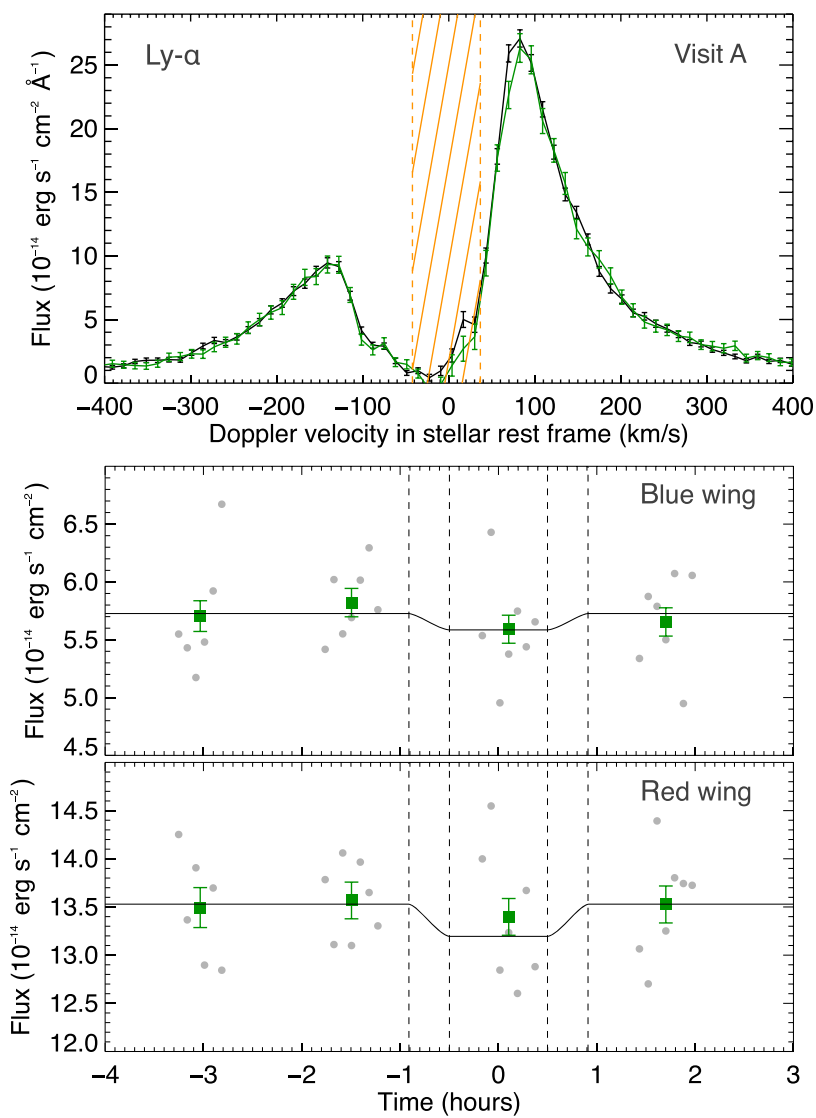

Figure 6. Top panel: Stellar Lyman $\alpha$ line in Visit A, during the optical transit of HD 189733b (green spectrum) and averaged over pre- and posttransit exposures (black spectrum). No variations are detected. The dashed orange region is too contaminated by geocoronal emission to be studied. Bottom panels: Lyman $\alpha$ flux integrated over the entire blue and red wings of the line, with the same codes as in Fig. 5.
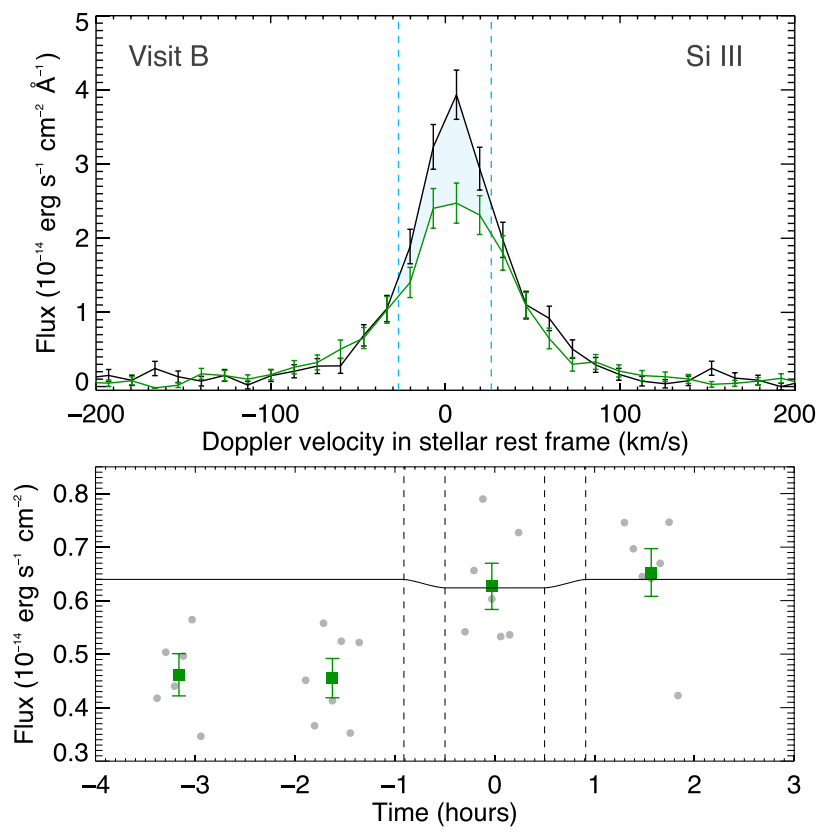

Figure 7. Si III line variations in Visit B. Same description as in Fig. 5, except that the stellar line profile is averaged over orbits 3 and 4 (black) and over orbits 1 and 2 (green). 


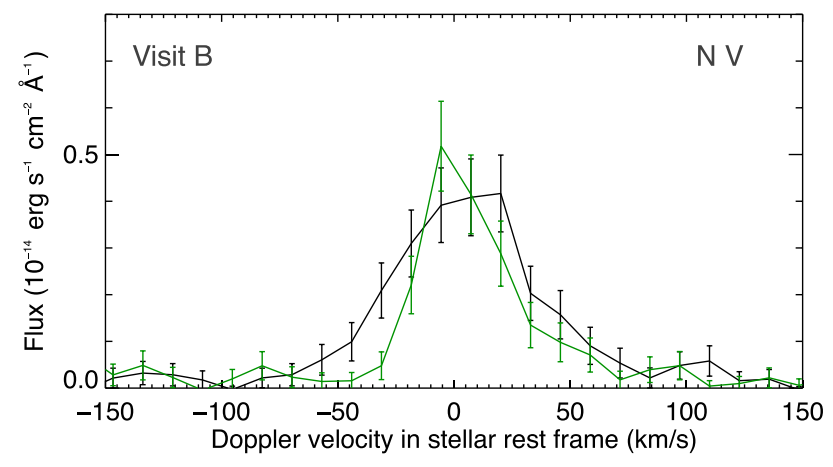

Figure 8. N v $\lambda 1243$ line variations in Visit B. Same description as in Fig. 7.

pre-transit orbits as reported in Bourrier et al. (2013). The line is too faint to determine precisely the spectral ranges of these variations, but they appear to be located in the wings (Fig. 8). We consider orbits 3 and 4 to be most representative of the quiescent $N \vee \lambda 1243$ stellar line, as it is similar to that of quiescent lines in other visits, and its flux is consistent with half that in the $\mathrm{N} V \lambda 1239$ line $(0.47 \pm 0.04)$, as expected from the ratio of the lines oscillator strengths in an optically thin medium. In contrast the $\mathrm{N} v$ lines flux ratio in orbits 1 and 2 is significantly lower than half $(0.38 \pm 0.04)$.

No variations are found in the Lyman $\alpha$ line during the pretransit orbits 1 and 2, which were taken as reference. We recover the significant absorption signature identified during in-transit orbit 3 by Lecavelier des Etangs et al. (2012), Bourrier et al. (2013), with decrease in stellar flux by $14.1 \pm 3.6$ per cent within -220.3 to $-128.1 \mathrm{~km} \mathrm{~s}^{-1}$ (Fig. 9). Subtracting the 2.4 per cent absorption by the UV atmospheric continuum (assumed to be the same as measured in the optical by Baluev 2015, see Section 3.6) yields an excess absorption of $11.7 \pm 3.6$ per cent by the exosphere of neutral hydrogen surrounding the planet. The flux decrease at the peak of the red wing in orbit 3 is marginal $(6.7 \pm 2.7$ percent within 69.3 to $122.0 \mathrm{~km} \mathrm{~s}^{-1}$ ), even more so when correcting for the planetary continuum. While this decrease cannot be considered by itself as a clear signature of the planetary atmosphere (Guo \& Ben-Jaffel 2016), it none-the-less occurs at the same time as the significant flux decrease in the blue wing, and we discuss its possible planetary origin in light of the new visits in Section 5. We do not detect any significant post-transit variation during orbit 4 (the blueshifted spectral range absorbed in orbit 3 yields a total variation of $5.2 \pm 3.9$ percent; see Lecavelier des Etangs et al. 2012 and Bourrier et al. 2013).

The temporal sampling of the Swift light curve (Fig. 9) does not allow a comparison between the X-ray variations and those measured in the FUV, but HD 189733 showed significant variability over the $\sim 28 \mathrm{~h}$ of observations obtained before, during, and after the transit. The count rate decreased over the duration of visit $\mathrm{B}$, and is interestingly lowest at the time of the planet transit. Furthermore a bright flare occurred about $8 \mathrm{~h}$ before ingress, as previously noted by Lecavelier des Etangs et al. (2012). Unlike the flare in the XMMNewton data (see next visits), there was no centroid shift towards either the M star companion or the background source, showing that the flare in Visit B arose from the planet host star.

\subsection{Visit C - 2013 May 9/10}

In Visit $\mathrm{C}$ no significant variations were found in any of the lines between orbits 1,3, and 4 . A flare occurred in orbit 2 in the Lyman $\alpha$ and Si III lines (Fig. 10). The flux increase appears to be maximum
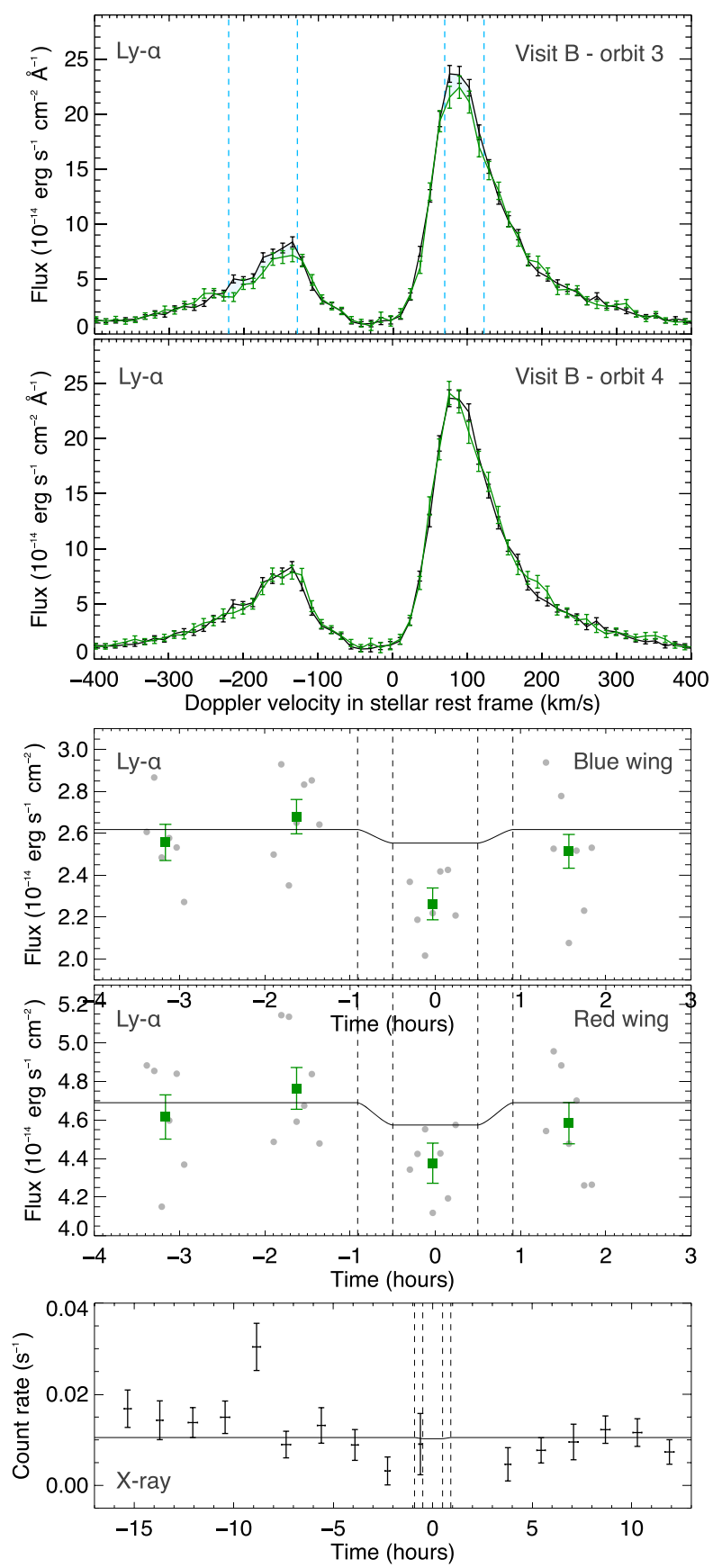

Figure 9. Top panels: Stellar Lyman $\alpha$ line in Visit B, before the optical transit of HD 189733b (black spectrum), during (green spectrum, top subpanel), and after (green spectrum, bottom subpanel). Middle panels: flux integrated over the spectral ranges absorbed in orbit 3. Bottom panel: Swift/XRT X-ray light curve obtained at the time of the transit in Visit B. The horizontal error bars correspond to the exposure time of data binned into one point per snapshot visit. The model transit light curve was normalized to the out-of-transit data, excluding the flare at about $-9 \mathrm{~h}$.

in sub-exposures 7 to 9 , during the ingress of HD 189733b. It is possible that the flare began earlier in the Si III line, but the dispersion of the flux in individual sub-exposures makes it difficult to identify which ones exactly were affected. The flare increased the flux over the entire observed profiles of the Lyman $\alpha$ and Si III lines, although the combination of ISM absorption and instrumental convolution 

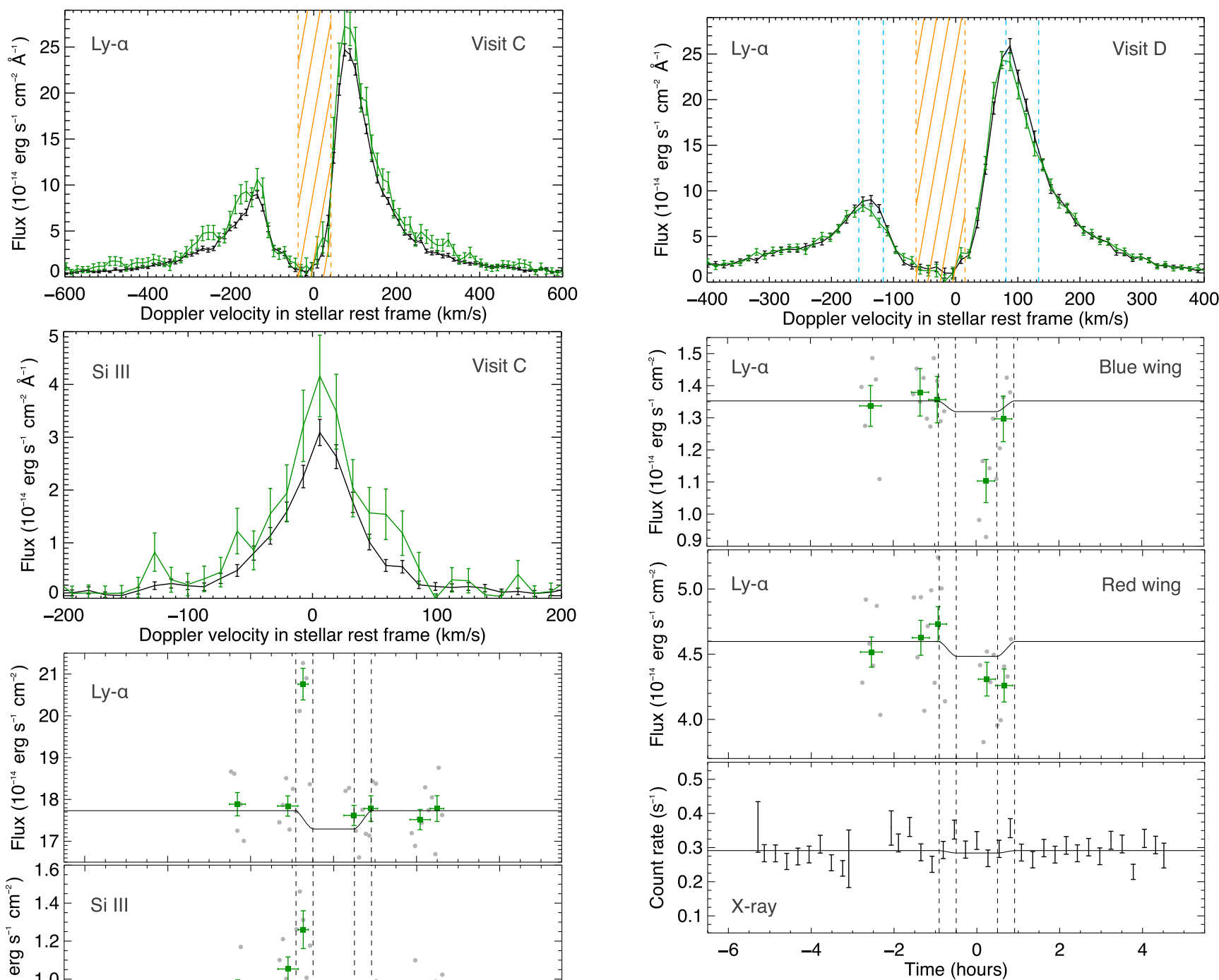

Figure 11. Top panel: Stellar Lyman $\alpha$ line in Visit D. Spectral ranges showing flux decreases in orbit 3 (green spectrum) compared to pre-transit orbits (black spectrum) as highlighted in blue. Middle panels: flux integrated over the spectral range absorbed in orbit 3 . The grey discs correspond to subexposures, binned by 6 or 5 (green squares). Bottom panel: XMM-Newton $\mathrm{X}$-ray light curve obtained at the time of the planet transit in Visit D. The model transit light curve was normalized to the out-of-transit data. Data contaminated by a flare from the $\mathrm{M}$ dwarf companion at about $-3 \mathrm{~h}$ has been excluded.

prevents us from assessing whether the core of the intrinsic Lyman $\alpha$ line was affected. Over the three most flaring sub-exposures, the Si III line increases by $44.9 \pm 12.5$ percent within $\pm 90 \mathrm{~km} \mathrm{~s}^{-1}$, and the Lyman $\alpha$ line increases by $21.1 \pm 4.2$ per cent over its blue wing and $16.3 \pm 2.7$ per cent over its red wing (between the airglow boundaries and $\pm 400 \mathrm{~km} \mathrm{~s}^{-1}$ ).

The Lyman $\alpha$ and Si III lines do not appear redshifted during the flare, as is sometimes the case for chromospheric and transition region lines (e.g. Pillitteri et al. 2015; Youngblood et al. 2017). We see no sign of the flare in the $\mathrm{NV}$ doublet, even though an increase on the order of that in the Si III line would have been detected. We also see no indication of the flare in the $\mathrm{O} V$ and Fe XII lines, although their faintness might prevent us from detecting such variations. The soft X-ray count rate is lower at the beginning of the 

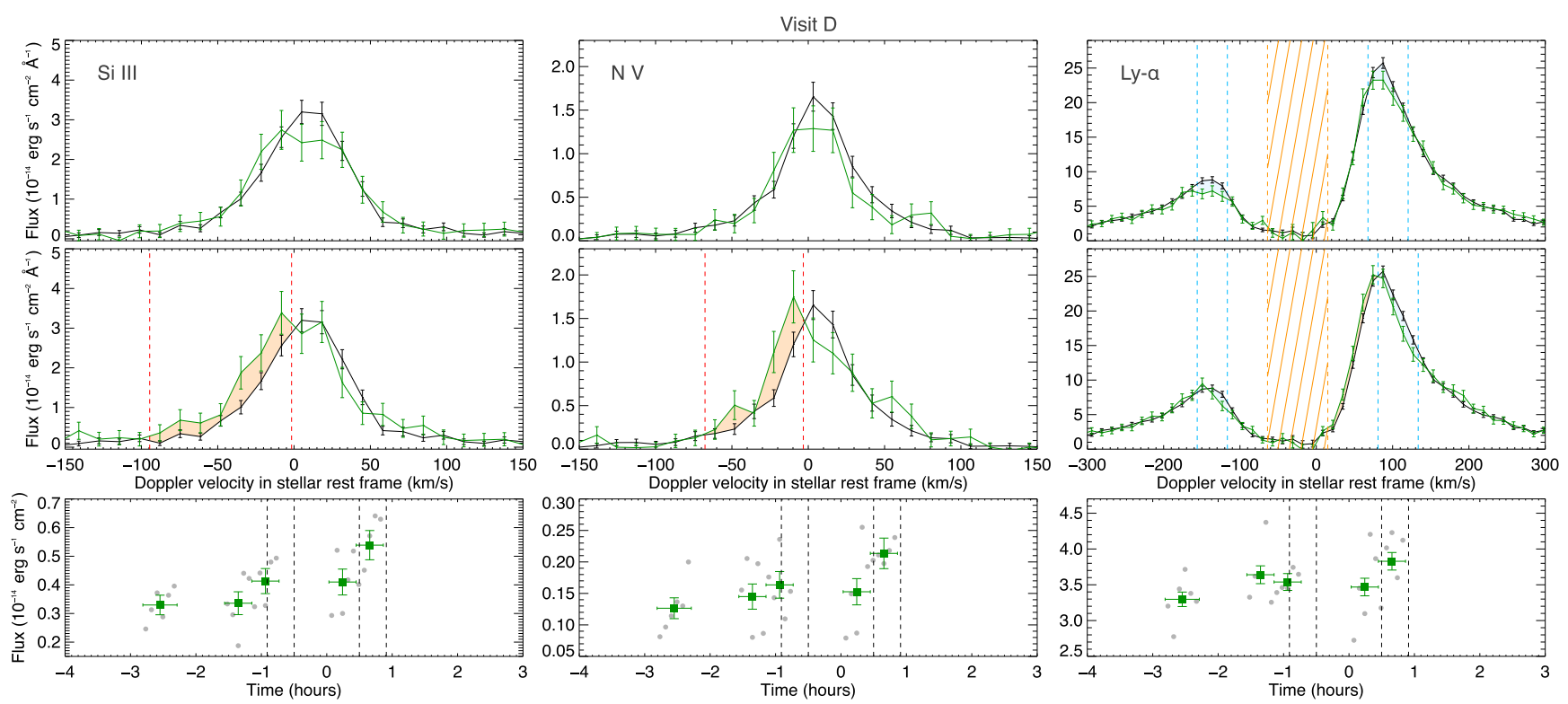

Figure 12. Variations in the Si III line (left-hand column), N V doublet (middle column), and Lyman $\alpha$ line (right-hand column) in Visit D. The green spectra show the lines during the first half (top row) and the second half (middle row) of orbit 3. Their comparison with the quiescent stellar lines (black spectra) reveals simultaneous flux increases during egress (highlighted as red regions). The blue regions highlight the absorbed spectral ranges in the Lyman $\alpha$ line. Bottom panels show the temporal evolution of the flux integrated over the flaring spectral regions. The green squares show sub-exposures (grey discs) binned by 6 or 5 .

visit but stabilizes after about $-3 \mathrm{~h}$, and shows no evidence for the flare (Fig. 10).

FUV-only flares have previously been observed in G-type stars and M dwarfs (Mitra-Kraev et al. 2005; Ayres 2015; Loyd et al. 2018). Flares result from reconnections occurring in magnetic structures. These reconnections accelerate electrons along the reconnecting field lines. When the electron beam impacts the dense lower atmosphere of the star, it rapidly heats the gas which expands to fill the reconnecting loop, producing the soft X-ray emission of the flare. The total energy available for this depends on the free magnetic energy available in the reconnecting loop. Loops with a greater reservoir of free energy can produce more energetic, higher temperature flares. Loyd et al. (2018) suggest that UV-only flares may be the result of reconnections in smaller magnetic structures that are only capable of heating the stellar transition region and chromosphere, whereas larger structures may release enough energy to drive hotter X-ray emitting plasma up into the corona. This scenario would be consistent with the relatively low amplification factors measured in HD 189733 Lyman $\alpha$ and Si III lines, and the non-detection of the flare in the higher energy lines and X-rays. The stronger amplification in the Si III line suggests that the energy released by the flare peaks at lower temperatures.

The observed flare is characterized by an abrupt rise in the Lyman $\alpha$ line and a longer rise in the Si III line (Fig. 10). While our observations do not cover the decay phase, the flux in both lines appear to return to its quiescent level in a short time $(\sim 50 \mathrm{~min}$ at maximum). This behaviour is consistent with that of the two flares observed by Pillitteri et al. (2015) in the FUV, which had short duration of $1 \mathrm{~h}$ and $400 \mathrm{~s}$ maximum, and also showed a larger flux increase in the Si III line compared to the NV doublet. On the other hand, lower optical chromospheric lines observed during a flare of HD 189733 with UVES showed a long decay after the initial short rise phase (Czesla et al. 2015; Klocová et al. 2017). These differences between optical and FUV lines likely traces a different behaviour between the lower and upper chromosphere of HD 189733 during flares.

\subsection{Visit D - 2013 November 3}

In Visit D no significant variations were found in any of the lines between orbits 1 and 2 . In orbit 3 the $\operatorname{Lyman} \alpha$ line shows two significant flux decreases (Fig. 11), in the blue wing $\left(13.2 \pm 4.4\right.$ percent between -156.1 and $\left.-116.5 \mathrm{~km} \mathrm{~s}^{-1}\right)$ and in the red wing $\left(7.6 \pm 2.5\right.$ per cent between 80.8 and $\left.133.6 \mathrm{~km} \mathrm{~s}^{-1}\right)$. The flux does not vary significantly over the region in between these signatures $\left(-5.7 \pm 3.0\right.$ percent between -116.5 and $80.8 \mathrm{~km} \mathrm{~s}^{-1}$ minus the airglow). These absorption signatures are reminiscent of those detected in Visit B: they occur during the planetary transit, have consistent absorption depths, and are located within similar spectral regions (although the blue wing signature in Visit D is less blueshifted than in Visit B). While the Visit D red wing signature remains stable during transit, the blue wing signature is deeper at mid-transit than its counterpart in Visit B $(21.3 \pm 5.7$ per cent in the first half of orbit 3) and disappears during egress (Fig. 11).

We caution that the red wing signature has only marginal transit depth when accounting for the planetary continuum. Furthermore, we found that the Lyman $\alpha$ flux in between the airglow and the red wing signature in fact increases sharply at egress during the second half of orbit 3 (by $16.0 \pm 5.5$ per cent within 15.0 to $67.8 \mathrm{~km} \mathrm{~s}^{-1}$ ). A simultaneous increase in flux occurs in the blue wings of the Si III line $(53.0 \pm 17.5$ per cent $)$ and $\mathrm{N}$ V doublet $(52.2 \pm 21.0$ per cent $)$, as can be seen in Fig. 12. These variations might be correlated and trace the onset of a flare in the stellar chromosphere, making it difficult to determine the exact properties of the Lyman $\alpha$ transit signatures.

There are no significant variations in the soft X-rays emitted by HD 189733 during Visit D, in particular during egress (Fig. 12), 


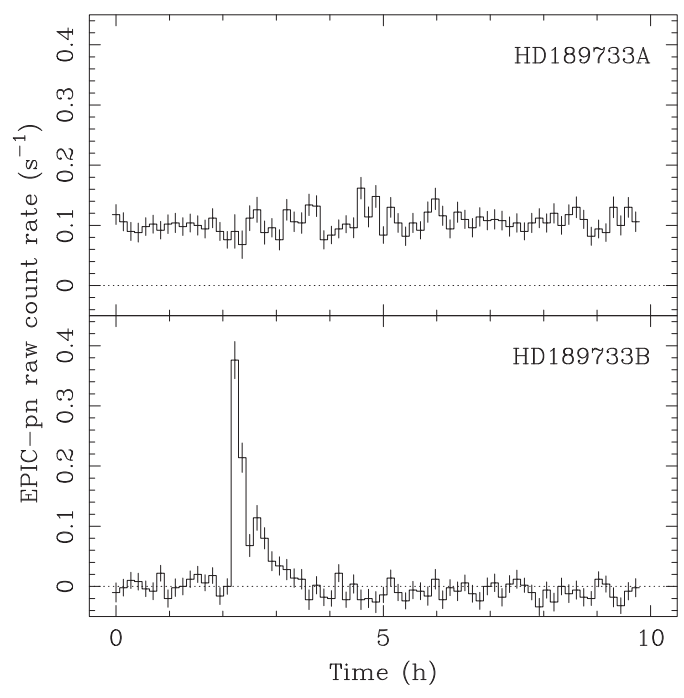

Figure 13. Comparison of the count rates for HD 189733 and its M star companion HD189733B in Visit D. Time is relative to the start of the X-ray observations. This clearly shows the flare is from the companion star.

although the example of Visit $\mathrm{C}$ shows that flares from this star can be limited to FUV lines.

We note that a strong flare did occur in the X-ray light curve about $3 \mathrm{~h}$ before mid-transit. Fig. 13 shows the separate X-ray count rates from each component of the system separately, obtained using the method described in Section 2.3. This clearly shows the flare to have been from the M dwarf companion HD 189733B, which calls in question the conclusion by Poppenhaeger et al. (2013) that it is inactive. Positional analysis confirms that the $\mathrm{M}$ dwarf is the origin of the flare, as the X-ray centroid is seen to shift to its position during the flare. Fig. 4 highlights this, where the $0.15-1.0 \mathrm{keV}$ image is dominated by emission from the position of primary star (leftmost panel) at all times except during the flare, when it shifts to the companion (second panel from the left). The flare is also seen originating from the M dwarf in the ultraviolet with the XMM OM (fourth panel of Fig. 4). The time period of the flare, which was covered by the first orbit of the HST visit, has been excluded in Fig. 12 and from the rest of the analysis. We do not see any evidence for the flare in STIS 2D images. We note that even in a case where HD 189733 B would enter the $52 \times 0.1$ arcsec-wide slit used in this visit, its spectrum would be located about 420 pixels from the spectrum of HD 189733 and would thus not contaminate its extraction. The variations in FUV lines discussed above thus arise from the primary HD 189733 or its planetary companion.

\subsection{Visit E - 2013 November 21}

The Lyman $\alpha$ line shows no significant variations in orbits 1 and 2. In orbit 3 both wings of the line show clear signatures, decreasing by $13.7 \pm 2.0$ percent within 75.8 and $181.2 \mathrm{~km} \mathrm{~s}^{-1}$, and by $10.6 \pm 2.7$ percent within -319.0 and $-108.3 \mathrm{~km} \mathrm{~s}^{-1}$ (Fig. 14). Strong flux decreases are also measured at larger velocities in both wings $(21.6 \pm 6.8$ percent within -503.3 and $-358.4 \mathrm{~km} \mathrm{~s}^{-1} ; 33.1 \pm 7.7$ per cent within 352.1 and $404.9 \mathrm{~km} \mathrm{~s}^{-1}$; $46.2 \pm 8.2$ percent within 510.0 and $589.1 \mathrm{~km} \mathrm{~s}^{-1}$ ). Even though the observed Lyman $\alpha$ line does not trace directly the intrinsic stellar line because of the combination of ISM absorption and instrumental convolution, we note that the decrease is on the same order ( $\sim 12$ percent) for the main signatures in the blue and red
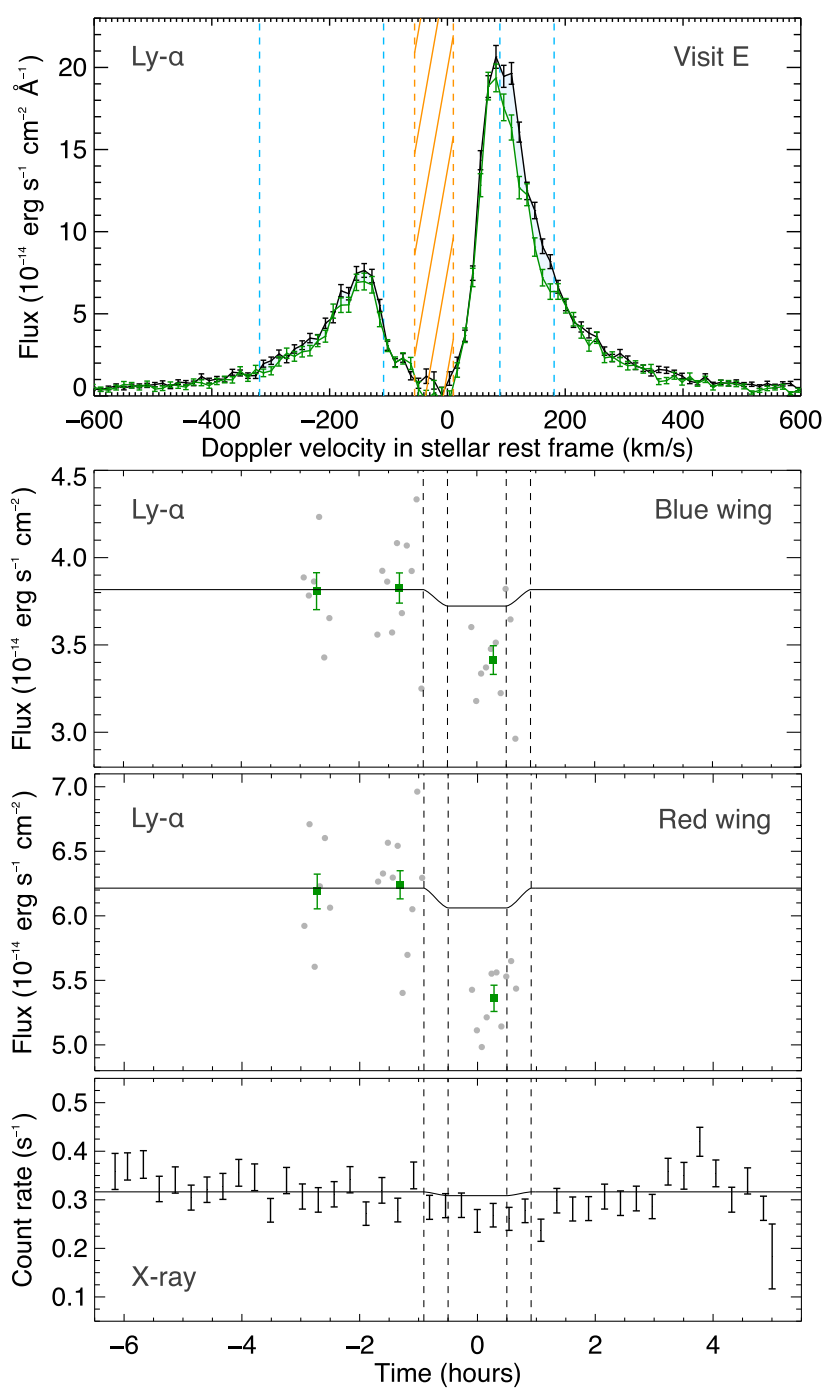

Figure 14. Top panel: Stellar Lyman $\alpha$ line in Visit E. Spectral ranges showing the clearest flux decreases in orbit 3 (green spectrum) compared to pre-transit orbits (black spectrum) as highlighted in blue. Middle panels: flux integrated over the spectral ranges shown in the top panel. The grey discs correspond to sub-exposures, green squares to orbit-averaged exposures. Bottom panel: XMM-Newton X-ray light curve obtained at the time of the planet transit in Visit E. The model transit light curve was normalized to the out-of-transit data.

wings of the line, and that the core of the observed line remains stable $\left(-2.6 \pm 3.5\right.$ per cent within -108.3 and $75.8 \mathrm{~km} \mathrm{~s}^{-1}$, airglow excluded).

Interestingly the soft X-ray count rate drops in the same time window as the Lyman $\alpha$ flux, before increasing suddenly about $3 \mathrm{~h}$ after mid-transit (Fig. 14). This tentatively suggests that the FUV flux decrease has a counterpart in the X-ray (Section 5).

No significant variations are detected in the Si III and the bright $\mathrm{NV} \lambda 1239$ lines. The NV $\lambda 1243$ line shows a similar flux between orbits 2 and 3, but is nearly twice brighter in orbit 1 (by $81.2 \pm 24.8$ per cent). Surprisingly the N v $\lambda 1243$ flux in orbit 1 and $2+3$ are respectively $0.670 \pm 0.079$ and $0.370 \pm 0.036$ times that of the average flux in the N V $\lambda 1239$ line, suggesting that none of the exposures is representative of the quiescent stellar line. This is further supported by the reconstruction performed in Section 4.4, which showed that the $\mathrm{NV} \lambda 1243$ line in either orbit 1 or orbit 
$2+3$ is not well fitted with the properties derived for the $N \vee \lambda 1239$ intrinsic line. While we do not know the origin of the large flux variation in the $\mathrm{N} v \lambda 1243$ line, its average over orbits 1,2 , and 3 likely best represents the quiescent stellar line, as it is about half as bright as the $\mathrm{N} v \lambda 1239$ line $(0.47 \pm 0.04)$ and is well fitted with its derived properties.

\subsection{Continuum light curves}

In previous sections, we identified spectra displaying strong flux variations in emission lines that could be caused by the star or by clouds of atoms and ions surrounding the planet. Other spectra showing no detectable variations were considered as representative of the quiescent stellar lines. However, during the transit these spectra are still absorbed by the planetary UV atmospheric continuum, which we sought to measure. We assumed a grey absorber yielding the same opacity at all wavelengths within a given stellar line, but varying with the spectral region. We thus fitted independent transit light curves to the flux integrated over the Lyman $\alpha$, Si III, and the cumulated Nv lines, using the EXOFAST routines (Mandel \& Agol 2002; Eastman, Gaudi \& Agol 2013). Given the precision and temporal coverage of our data we assumed a uniform stellar disc with no limb-darkening or brightening. Visit E was not included in the fit to the Lyman $\alpha$ and $\mathrm{NV}$ light curves, as its in-transit exposures show strong variability in these lines (Section 3.5). We fitted the planet-to-star radii ratio $R_{\mathrm{p}}^{\text {line }} / R_{*}$, and fixed all other system properties to the values given in Table 1 .

We sampled the posterior distributions of the model parameters using the Markov-Chain Monte Carlo (MCMC) PYTHON software package EMCEE (Foreman-Mackey et al. 2013). The best-fitting transit light curves in the region of each line, shown in Fig. 15, correspond to $R_{\mathrm{p}}^{\mathrm{Ly}-\alpha} / R_{\star}=0.092_{-0.053}^{+0.039}, R_{\mathrm{p}}^{\mathrm{Siiii}} / R_{\star}=0.29_{-0.12}^{+0.08}$, $R_{\mathrm{p}}^{\mathrm{Nv}} / R_{\star}=0.15 \pm 0.10$. These values reveal a marginal transit detection in the $\mathrm{Si}$ III line $(2.4 \sigma)$, but are not significantly different from $0(<3 \sigma)$ and consistent with the optical transit $\left(R_{\mathrm{p}} / R_{\star}=0.1571 \pm 0.0004\right.$, corresponding to a transit depth of 2.4 percent; Baluev 2015). Fitting a common transit model to the combined Lyman $\alpha$, Si III, and $\mathrm{NV}$ fluxes yield $R_{\mathrm{p}}^{\mathrm{FUV}} / R_{\star}=0.104_{-0.049}^{+0.036}$, similarly consistent with the optical transit and different from 0 by only $2 \sigma$. The present data are therefore not precise enough to measure the atmospheric continuum of HD 189733 b in the FUV.

\section{ANALYSIS OF THE HIGH-ENERGY STELLAR SPECTRUM}

We used the quiescent X-ray and FUV spectra identified in Section 3 to analyse the high-energy spectrum of HD 189733 and its evolution over the different observing epochs. Spectra obtained during the planetary transit were corrected for the planetary continuum absorption, fixed to 2.4 per cent (see Section 3.6).

\subsection{Retrieval of the stellar Lyman $\alpha$ line and ISM properties}

Knowledge of the intrinsic stellar Lyman $\alpha$ line profile is important to our understanding of the stellar chromosphere, its extreme ultraviolet (EUV) emission, and its impact on the planetary atmosphere. We reconstructed the theoretical line profiles in each epoch, following the same procedure as in, e.g. Bourrier et al. (2015, 2017). A model profile of the intrinsic stellar line is absorbed by hydrogen and deuterium in the ISM, convolved with STIS line

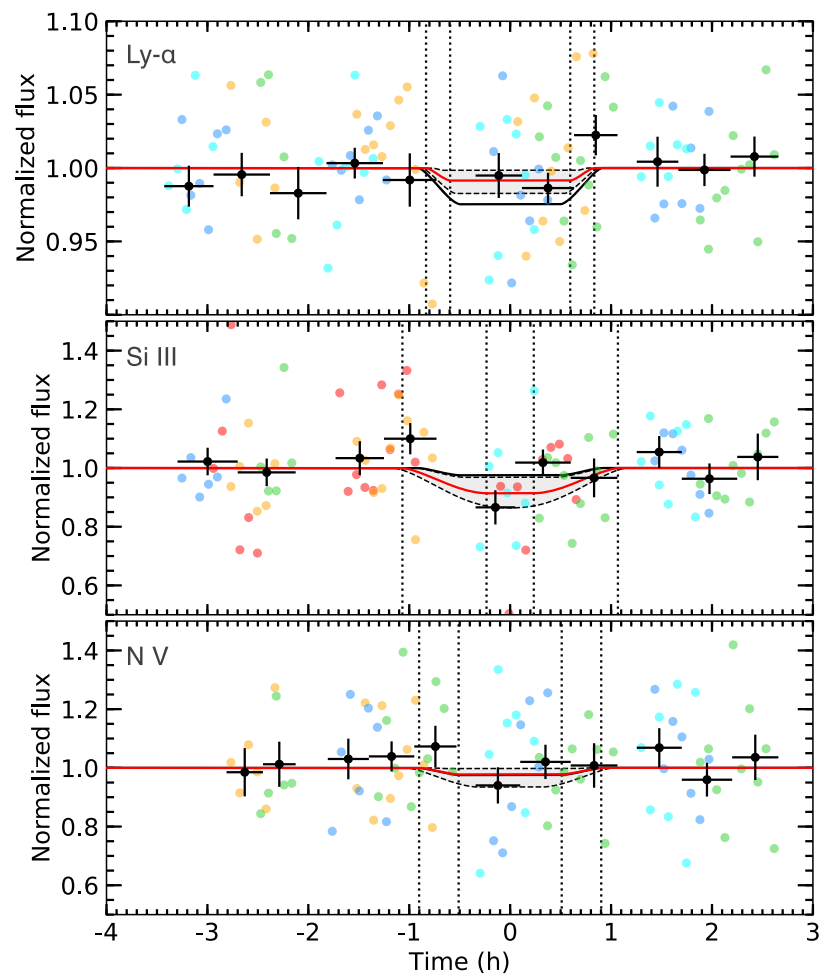

Figure 15. Transit light curves of HD 189733b in the Lyman $\alpha$ (top), Si III (middle), Nv (bottom) lines. The flux has been integrated over the largest spectral range showing no strong variations in each visit, and only visits with stable sub-exposures both out- and in-transit have been fitted with a transit model. The best-fit is shown as a red line, with the corresponding contact times highlighted with dotted vertical lines. The grey bands delimit the $1 \sigma$ envelopes of the best-fitting models. The solid black line shows the transit light curve with the planet optical radius in front of a uniform disc, for comparison. Sub-exposures are coloured in deep blue for Visit A, cyan for Visit B, green for Visit C, orange for Visit D, and red for Visit E. The black points show binned sub-exposures.

spread function (LSF, as derived by Bourrier et al. 2017), and fit to the quiescent Lyman $\alpha$ lines in each visit. These master spectra were built by co-adding the flux over the spectral ranges identified as stable in each exposure. The model is oversampled in wavelength, and rebinned over the STIS spectral table after convolution. The comparison between observed and theoretical data was performed between -400 and $400 \mathrm{~km} \mathrm{~s}^{-1}$ (defined in the star rest frame), excluding the airglow-contaminated spectral ranges (Fig. 2). We sampled the posterior distributions of the master spectra parameters using EMCEE, and derived their best-fitting values and uncertainties using the same method as in Bourrier et al. (2018b).

We performed preliminary simulations to determine the best model for the intrinsic stellar line, using the BIC as merit function. This revealed three interesting features: (i) the intrinsic line is best fitted with a double-peaked Voigt profile (compared to other typical line profiles with a single peak or Gaussian components, e.g. Wood et al. 2005; Youngblood et al. 2016), confirming previous findings by Bourrier et al. (2013); (ii) there is no significant difference in the velocity separation between the two peaks (assumed to be identical) between the different visits; (iii) in each visit, the intrinsic stellar line is well centred on the Lyman $\alpha$ transition wavelength in the star rest frame. The final theoretical intrinsic line was thus modelled as two Voigt profiles with the same total flux, temperature (assuming pure thermal broadening), and damping parameter. These three free 

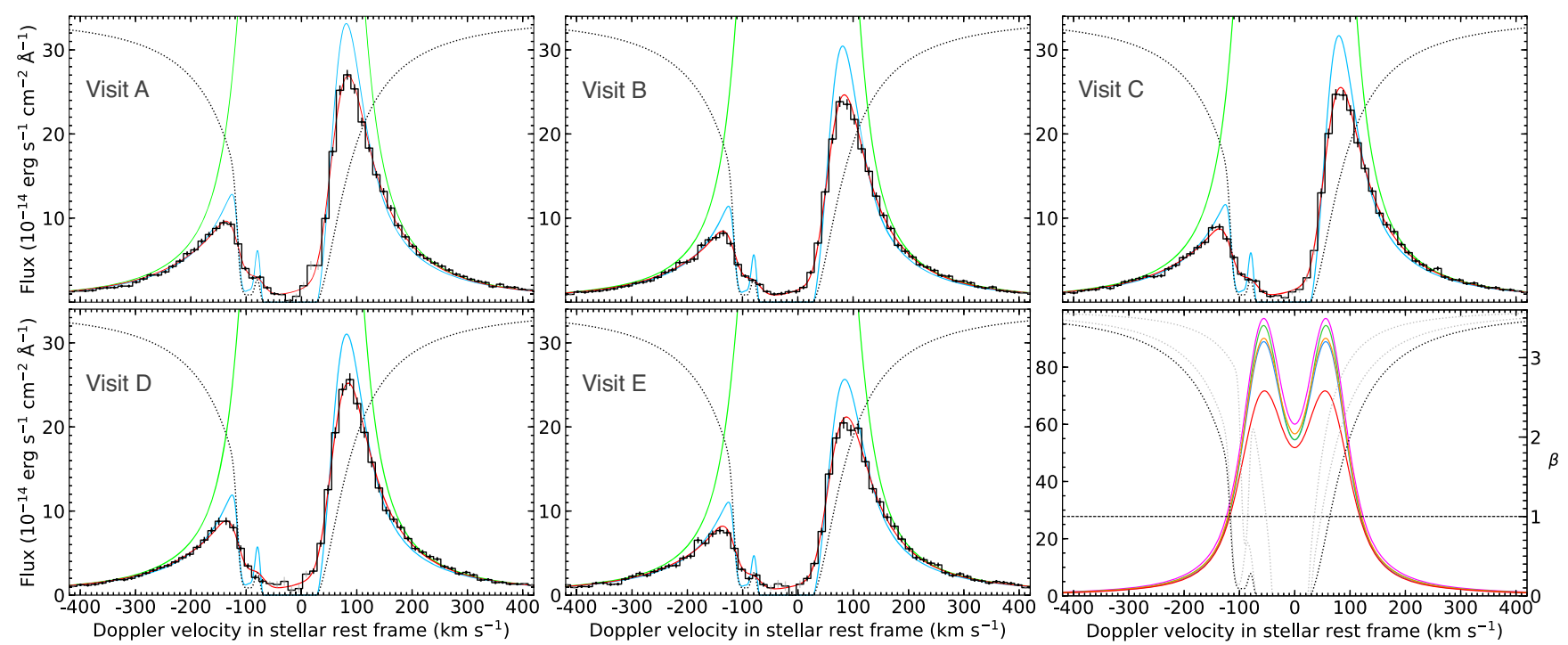

Figure 16. Quiescent Lyman $\alpha$ line profiles of HD 189733. The black histograms show observed spectra, fit over points with black error bars. The green lines are the best fits for the intrinsic stellar line profiles in each visit, at Earth distance. They yield the blue profile after absorption by the interstellar medium, and the red profile after further convolution with the STIS LSF. The bottom right-hand panel shows a comparison of the intrinsic stellar line profiles between the visits (coloured in purple, blue, green, orange, and red from Visit A to E), with the corresponding radiation pressure to stellar gravity $\beta$ ratio reported on the right axis. The total ISM absorption profile is plotted as a dotted black line in all panels (scaled to the panel vertical range), with the absorption from individual ISM components plotted shown as dotted grey lines in the bottom right-hand panel.

parameters are specific to each visit. The two Voigt profiles are placed at the same distance from the Lyman $\alpha$ transition wavelength in the star rest frame, and their velocity separation is a free parameter common to all visits.

The theoretical absorption profile of the ISM along the line of sight is common to all visits, and defined by its column density of neutral hydrogen $\log _{10} N_{\text {ISM }}\left(\mathrm{HI}\right.$ ), its temperature $T_{\text {ISM }}$, and turbulent

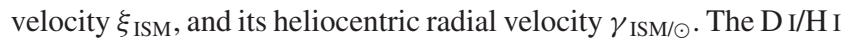
ratio was set to $1.5 \times 10^{-5}$ (e.g. Hébrard \& Moos 2003; Wood et al. 2004; Linsky et al. 2006). The spectral resolution of the STIS data and the small difference in mass between hydrogen and deuterium prevent us from constraining both the temperature and turbulent velocity, and the latter was thus fixed to a constant value. The LISM kinematic calculator ${ }^{2}$ (Redfield \& Linsky 2008) predicts that the line of sight (LOS) towards HD 189733 crosses the Mic $\left(T_{\text {Mic }}=\right.$ $9900 \pm 2000 \mathrm{~K}, \xi_{\text {Mic }}=3.1 \pm 1.0 \mathrm{~km} \mathrm{~s}^{-1}, \gamma_{\text {Mic } / \odot}=-22.2 \pm$ $\left.1.3 \mathrm{~km} \mathrm{~s}^{-1}\right)$, Eri $\left(T_{\text {Eri }}=5300 \pm 4000 \mathrm{~K}, \xi_{\text {Eri }}=3.6 \pm 1.0 \mathrm{~km} \mathrm{~s}^{-1}\right.$, $\left.\gamma_{\mathrm{Er} / \odot}=-15.9 \pm 1.0 \mathrm{~km} \mathrm{~s}^{-1}\right)$, and Aql $\left(T_{\mathrm{Aql}}=7000 \pm 2800 \mathrm{~K}\right.$, $\xi_{\mathrm{Aql}}=2.1 \pm 0.6 \mathrm{~km} \mathrm{~s}^{-1}, \gamma_{\mathrm{Aql} / \odot}=-18.6 \pm 1.0 \mathrm{~km} \mathrm{~s}^{-1}$ ) clouds. In a first step, we assumed that a single cloud contributes to the ISM opacity along the LOS, and we fixed $\xi_{\text {ISM }}$ to the error-weighted mean of the three clouds turbulent velocities. This led to an ISM cloud with $\gamma_{\mathrm{ISM} / \odot}=-21.3 \pm 0.5 \mathrm{~km} \mathrm{~s}^{-1}$, which suggests that the Mic cloud is the dominant ISM opacity source. However, the single-cloud model yields $T_{\mathrm{ISM}}=15365 \pm 500 \mathrm{~K}$, which is much larger than temperatures expected for the local ISM (Redfield \& Linsky 2008), in particular in the direction of HD 189733. We thus performed the final fit using a two-cloud ISM model. The turbulent velocity of component A was fixed to that of the MIC cloud, and the turbulent velocity of component $\mathrm{B}$ was fixed to the error-weighted mean of the Eri and Aql cloud values. Other properties were let free to vary independently for each component.

\footnotetext{
${ }^{2} \mathrm{http}: / /$ sredfield.web.wesleyan.edu/
}

Best-fitting models are shown in Fig. 16. They yield a good $\chi^{2}$ of 277 for 260 degrees of freedom $\left(\chi_{\mathrm{r}}^{2}=1.07\right.$, with 282 data points and 22 free parameters). Properties of interest for the Lyman $\alpha$ line and ISM are given in Table 3. The double-cloud ISM model improves the $\chi^{2}$ with no change in the BIC compared to the single-cloud ISM model $\left(\chi^{2}=292, \mathrm{BIC}=400\right)$. The properties of component $\mathrm{A}\left(T_{\mathrm{A}}=13074_{-1083}^{+1137} \mathrm{~K}, \gamma_{\mathrm{A} / \odot}=-23.7_{-1.7}^{+1.2} \mathrm{~km} \mathrm{~s}^{-1}\right)$ are more consistent with those of the Mic cloud, while component $\mathrm{B}$ has a temperature in between those of the Eri and Aql clouds but is more redshifted $\left(T_{\mathrm{B}}=5755_{-2802}^{+2910} \mathrm{~K}, \gamma_{\mathrm{B} / \odot}=-10.8_{-2.4}^{+2.2} \mathrm{~km} \mathrm{~s}^{-1}\right)$. The column densities of both components (Table 3 ) are in the range expected for a star at a distance of $19.8 \mathrm{pc}$ (fig. 14 in Wood et al. 2005).

\subsection{Analysis of the quiescent FUV stellar lines}

Stellar lines in the FUV provide useful information about the structure and emission of the chromosphere and transition region. We derived the properties of the Si III, O v, Nv, and Fe XII lines following the same approach as for the Lyman $\alpha$ line (Section 4.1). Models were fitted to the quiescent spectra, averaged over exposures identified as stable in each visit (Section 3). The average of the quiescent spectra over all visits further revealed the faint Si II $\lambda 1197.4$ line. Voigt profiles were found to better model the bright Si III and NV lines, while Gaussian profiles are sufficient for the Si II, O V, and Fe XII lines. We assumed that the model lines are thermally broadened, and allowed their centroid to vary in the star rest frame (defined by the GAIA radial velocity, assumed to trace the photosphere). We fitted a flat continuum level in the region of the Si II, Si III, and N V line, and a polynomial representing the Lyman $\alpha$ red wing in the region of the $\mathrm{O} v$ line. The Fe XII line, blended with the NV $\lambda 1239$ line, was fitted together with the $\mathrm{NV}$ doublet. We assumed a common temperature, damping parameter, and Doppler shift for the $\mathrm{N} v$ lines. 
Table 3. Properties derived from the fit to the HD 189733 stellar lines and the reconstructed XUV spectrum.

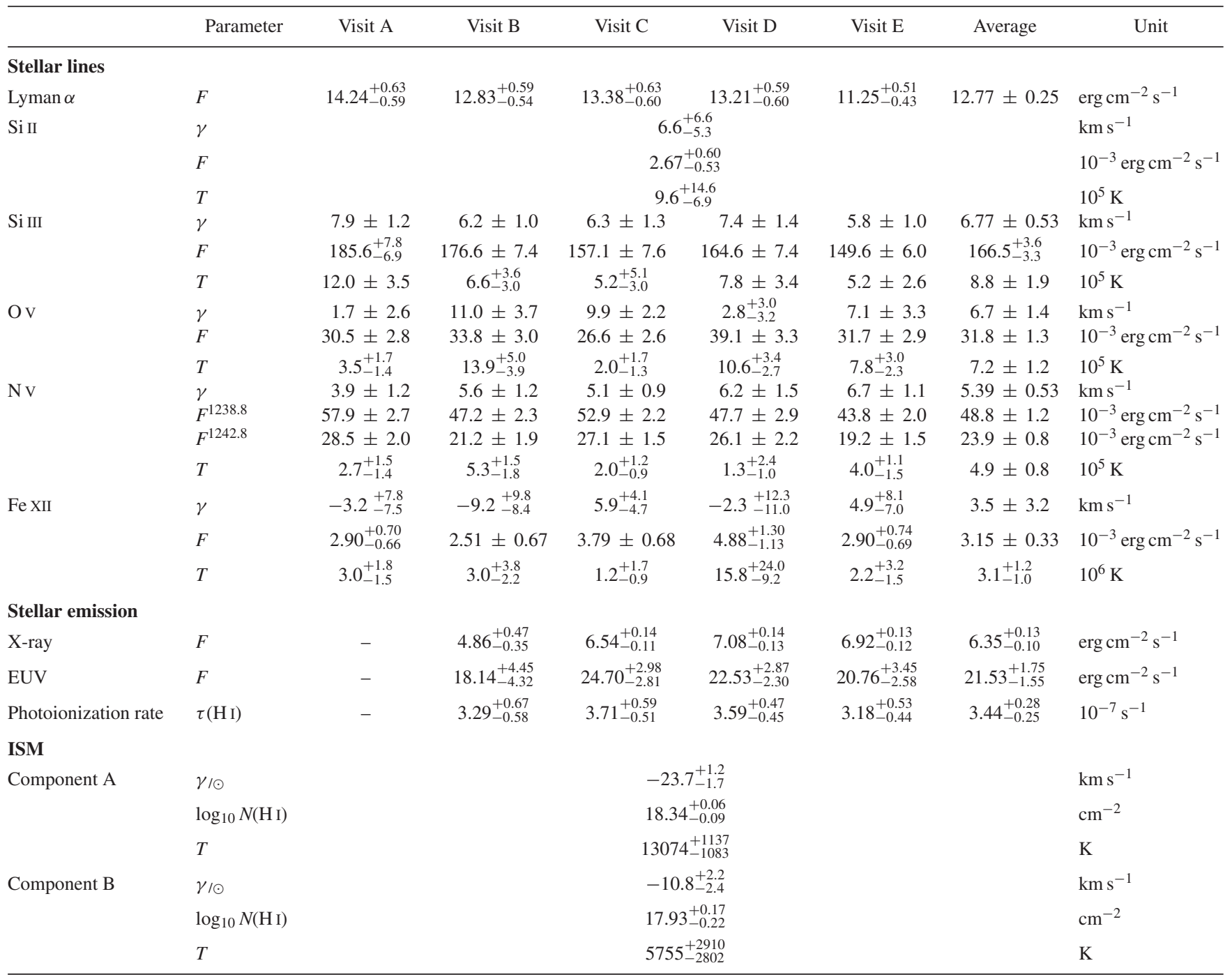

Note. $\gamma$ is the radial velocity of a model line centroid in the star rest frame, and $T$ its temperature. $F$ is the total flux at 1 au from the star, in the model FUV lines, in the synthetic EUV spectra $(62-912 \AA)$, and in the model X-ray spectra $(0.2-2.4 \mathrm{keV}=5.2-62.0 \AA) . \tau(\mathrm{HI})$ is the photoionization rate of neutral hydrogen atoms corresponding to the mean XUV spectrum at 1 au. $\gamma_{/ \odot}$ is the radial velocity of a model ISM cloud relative to the Sun, $\log _{10} N(\mathrm{HI})$ its column density of neutral hydrogen, and $T$ its temperature (assuming fixed turbulent broadening values, see the text).

The best-fitting properties for the quiescent lines in each visit, and for the lines averaged over all visits, are reported in Table 3. While the Lyman $\alpha$ line is well aligned in the star rest frame (Section 4.1), we detect significant redshifts for the Si III, N V, and OV lines (Fig. 17). This pattern is observed in the Sun (Achour et al. 1995; Peter \& Judge 1999) and other stars (e.g. Linsky et al. 2012), showing that the STIS spectra of HD 189733 are well calibrated and that the measured redshifts trace the structure of the transition region between the stellar chromosphere and corona. The amplitude and variation of HD 189733 ( $\mathrm{P}_{\text {rot }} \sim 8-10 \mathrm{~d}$ ) emission-line redshifts with formation temperature are consistent with those observed for stars rotating slower than $\sim 4 \mathrm{~d}$ (Linsky et al. 2012). Namely, the Lyman $\alpha$ line and chromospheric lines formed at low temperatures (e.g. Si II) do not show significant deviation from the photosphere velocity, while lines formed at $\log T$ $\gtrsim 4.5$ up to the transition region get increasingly redshifted. The most redshifted lines of HD 189733 are Si III, N V, and O V, formed at temperatures in between $\log T \sim 4.7-5.3$ that are expected to

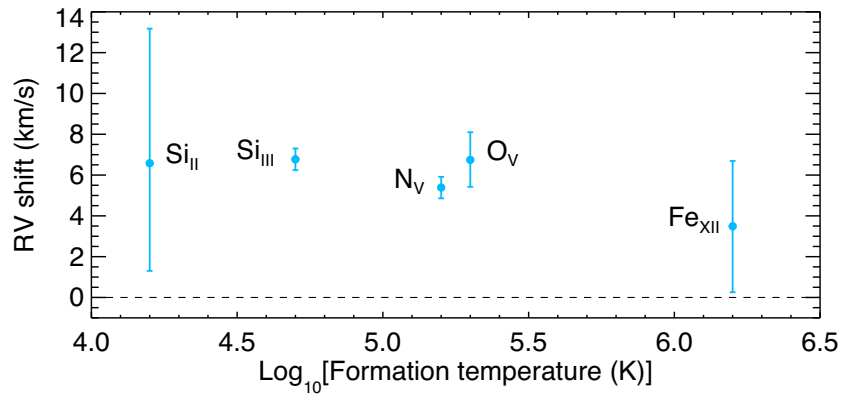

Figure 17. Shifts of HD 189733 emission lines relative to its photosphere radial velocity. Shifts have been derived from the quiescent lines averaged over all epochs. The Lyman $\alpha$ line is not shifted with respect to the photosphere. Transition wavelengths associated to each line were taken from the NIST Atomic Spectra Data base (Kramida, Ralchenko \& Reader 2016). Line formation temperatures are from the Chianti v.7.0 data base (Dere et al. 1997; Landi et al. 2012). 


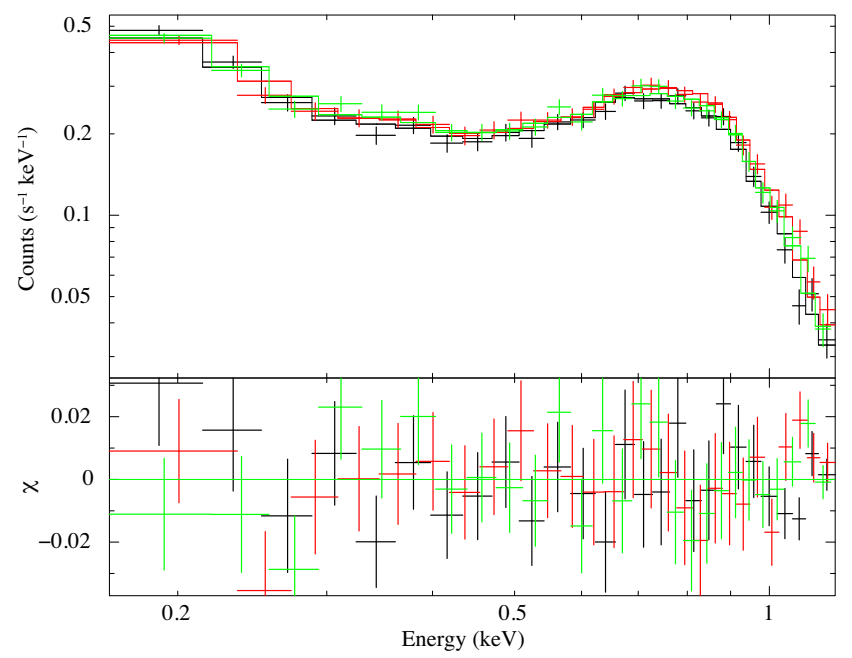

Figure 18. X-ray spectra for the $X M M$-Newton data sets (coloured points), together with their best-fitting model for the spectra of HD 189733 (coloured histograms), in the soft energy range uncontaminated by the background source. Visit $\mathrm{C}$ is in black, visit $\mathrm{D}$ is in red, and visit $\mathrm{E}$ is in green. The bottom panel shows the residual between the measured spectra and their fit.

yield the maximum redshifts (Linsky et al. 2012). Redshifts are then expected to decrease with rising temperature, and coronal lines can even display blueshifts for $\log T \gtrsim 5.7$. Although we do not have sufficient precision to draw a firm conclusion, the Fe XII line is consistent with being less redshifted (Fig. 17). This pattern of deviations from the photosphere velocity can be explained by the heating of gas in the upper chromosphere, which propagates upwards into the corona along the leg of a magnetic loop (emitting blueshifted lines), subsequently cools, and rains down along the other leg of the loop into the transition region (emitting redshifted lines). Interestingly the derived line temperatures (Table 3) are systematically larger than their expected formation temperatures (Fig. 17), which could possibly trace additional broadening due to the motion of the rising/falling gas. More information about this mechanism can be found in, e.g. Peter \& Judge (1999), Hansteen et al. (2010), Linsky et al. (2012) (see also Bourrier et al. 2018a for possible signatures of planet-induced coronal rain in the star $55 \mathrm{Cnc})$.

\subsection{Analysis of the stellar X-ray spectrum}

The XMM-Newton EPIC-pn spectra extracted in Section 2.3 for Visit C, D, and E were analysed using XSPEC 12.9.1p (Arnaud 1996). The X-ray spectra for these three visits, displayed in Fig. 18, are very similar in shape and flux. They are reasonably soft, typical of moderately active late-type stars a few Gyr old, including strong line emission between 0.6 and $0.9 \mathrm{keV}$ due primarily to oxygen and the L-shell transitions of iron.

We used APEC models (Smith et al. 2001) to fit the X-ray spectra, limiting the fit to energies below $1.2 \mathrm{keV}$ to avoid contamination by the background source (Section 2.3). We found that at least three temperature components were required to reproduce the spectra $\left(\mathrm{kT}_{1}=0.147_{-0.007}^{+0.059} \mathrm{keV}, \mathrm{kT}_{2}=0.345_{-0.032}^{+0.031} \mathrm{keV}\right.$, $\mathrm{kT}_{3}=0.724_{-0.090}^{+0.034} \mathrm{keV}$ ). Although we stress that this should be thought of as an approximation to a plasma with a continuous range of temperatures. Each temperature component was linked across the three observations, thereby forcing the spectral shape to remain the same. However, we did allow the total emission measures, and therefore fluxes, to change. An interstellar absorption term was included for completeness by using the TBABS model (Wilms, Allen \& McCray 2000), though its contribution to the results was negligible given the relatively close proximity of HD 189733 to Earth (Table 1).

Using fixed Solar abundances across all species (Caffau et al. 2011), we were not able to attain a statistically acceptable fit. Freeing up elements $(\mathrm{C}, \mathrm{N}, \mathrm{O}, \mathrm{Ne}, \mathrm{Fe})$ relevant for the first ionization potential (FIP) effect (e.g. Feldman 1992; Laming 2015) yielded a far superior fit. We obtained a coronal $\mathrm{Ne} / \mathrm{Fe}$ value of 7.9, a value in between quiet and very active K0/K1 stars (Wood, Laming \& Karovska 2012; Laming 2015). Additionally, we estimate from the coronal and photospheric abundances $F_{\text {bias }}=\log _{10}(\mathrm{X} / \mathrm{Fe})_{\text {cor }}-$ $\log _{10}(\mathrm{X} / \mathrm{Fe})_{\text {phot }}$, where $\mathrm{X}$ is abundance of the high FIP species being tested. Our final value of $F_{\text {bias }}$ is $0.65_{-0.07}^{+0.15}$, obtained by averaging across the four species we freed up, and is indicative of a relatively strong inverse FIP effect in HD 189733. This result disagrees with that of Poppenhaeger et al. (2013), who found an $F_{\text {bias }}$ of -0.41 in their fit to six Chandra observations when freeing up only $\mathrm{Ne}, \mathrm{O}$, and $\mathrm{Fe}$. However, our abundances for $\mathrm{N}$, which exhibits the greatest inverse FIP effect, and Fe are independently corroborated in the differential emission measure (DEM) fit to the high-excitation FUV lines in Section 4.4. Our resultant X-ray fluxes in the $0.2-2.4 \mathrm{keV}$ band are given in Table 3 and shown in Fig. 20.

We forced the fit to the Swift data in Visit B to have the same temperatures and abundances as the $X M M-N e w t o n$ fit, given the limited number of counts. The normalizations, and hence fluxes, were allowed to change. We fitted independently the spectra for the flaring and non-flaring times of HD 189733. In the $0.2-2.4 \mathrm{keV}$ band we derive a flux of $\left(4.86_{-0.35}^{+0.47}\right) \mathrm{erg} \mathrm{s}^{-1} \mathrm{~cm}^{-2}$ for the nonflaring spectrum at 1 au from the star, lower than the three $X M M-$ Newton epochs. In-flare, the flux rises to $\left(12.0_{-0.9}^{+3.9}\right) \mathrm{erg} \mathrm{s}^{-1} \mathrm{~cm}^{-2}$, unsurprisingly considerably higher than the quiescent flux in all three XMM-Newton observations.

\subsection{Reconstruction of the stellar EUV spectrum}

Most of the stellar EUV spectrum is not observable from Earth because of ISM absorption. We therefore reconstructed the entire XUV spectrum up to $1600 \AA$ using the DEM retrieval technique described in Louden, Wheatley \& Briggs (2017). This reconstruction is based on the quiescent X-ray flux for the coronal region (from the data shown in Section 3), and on the flux derived for the intrinsic FUV stellar lines (Section 4.2) for the chromosphere and transition region. The XUV spectra could thus be reconstructed for Visits B to $\mathrm{E}$.

Due to the high SNR of the X-ray spectra we chose not to use Chebyshev Polynomials to calculate the shape of the DEM's, instead using a regularized inversion approach, as in Hannah \& Kontar (2012), otherwise the technique is the same as described in Louden et al. (2017). We found that a regularization parameter of 100 gave the best balance between model complexity and fit to the data. Initially, the abundances were set to solar photospheric values (Caffau et al. 2011) for the UV lines and solar coronal (Schmelz et al. 2012) for X-ray flux. This resulted in a poor fit to the $\mathrm{O} v$ and $\mathrm{N} v$ lines, which are formed at a similar characteristic temperature of $\sim 10^{5.3} \mathrm{~K}$, and the Fe XII line, formed at $\sim 10^{6.3} \mathrm{~K}$. We then fit again, allowing the nitrogen and iron abundances to vary. We found consistently in the four visits that the best-fitting values for the nitrogen and iron abundances were respectively 3.8 and 0.6 times the values derived by Caffau et al. 2011. This is not an unexpected result, due both to the gross differences in abundances between stars, 

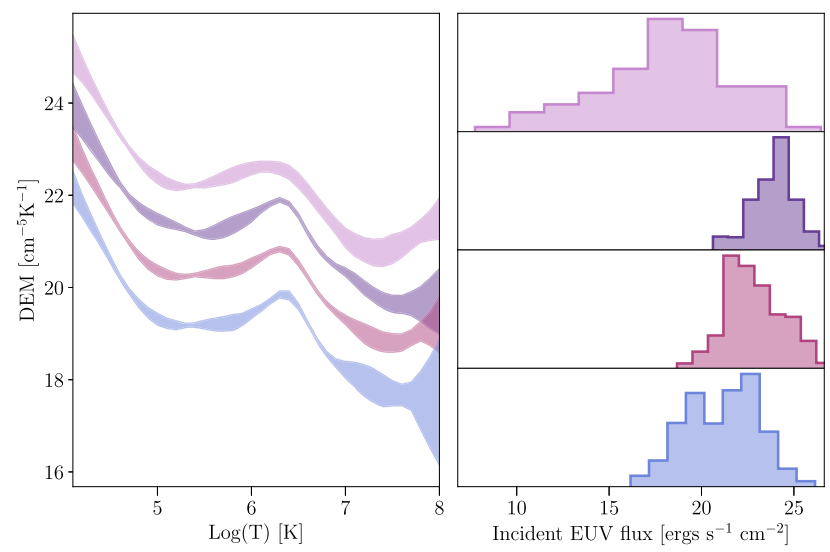

Figure 19. Left-hand panel: The $1 \sigma$ credible region for the DEM's for Visits B to E (from top to bottom) with offsets for clarity. The shape of the DEM remains consistent between the four visits, except for the very high temperatures where the constraints from the data are weaker. Right-hand panel: The posteriors of the EUV (62-920 ̊) flux at 1 au for the four nights, in the same order. The constraints are weakest in Visit B due to the lower SNR of the Swift data, demonstrating the necessity of wide coverage for accurate reconstructions.

and also the potential effects of the FIP and inverse FIP effects in modifying these abundances in the star's upper atmosphere. We then repeated the fit for each of the four visits with these values fixed to generate our final DEM and spectra. With the abundances thus modified the fits to the lines were significantly improved. The flux of each line was recovered on each night to within $1.5 \sigma$, and the $\mathrm{X}$-ray flux was recovered with consistent values to those found in Section 4.3. The final DEM's, as well as the posteriors for the total EUV fluxes at $1 \mathrm{au}$, are plotted in Fig. 19, with the corresponding best-fitting values given in Table 3 . The generated XUV spectra for the four visits are available online as machine readable tables.

The synthetic XUV spectra yield an average photoionization lifetime of about $33.6 \mathrm{~d}$ for neutral hydrogen at 1 au from HD 189733, which corresponds to $50 \mathrm{~min}$ at the orbital distance of HD $189733 \mathrm{~b}$ (details on the calculation can be found in Bourrier et al. 2017). Implications for the structure of the planetary exosphere are discussed in Section 5. The lifetime at 1 au ranges within its uncertainties between 29 and $43 \mathrm{~d}$ in Visit $\mathrm{C}$, which is close to the range $\sim 6.5$ 25 d derived by Bourrier \& Lecavelier des Etangs (2013) from the fit to the Lyman $\alpha$ transit in this visit. As the atmospheric mass-loss of neutral hydrogen correlates positively with its photoionization rate in this fit, the larger lifetimes we obtained suggest that the neutral hydrogen loss from HD $189733 \mathrm{~b}$ in Visit B is at the lower end of the range derived by Bourrier \& Lecavelier des Etangs (2013) $\left(\sim 10^{9} \mathrm{~g} \mathrm{~s}^{-1}\right.$ or lower).

\subsection{Temporal evolution of the stellar XUV spectrum}

Overall the quiescent emission of HD 189733 in the observed FUV lines remains quite stable over the $2 \mathrm{yr}$ and a half covered by our visits (Fig. 20). We note, however, two interesting features. First, low-temperature lines (Lyman $\alpha, \mathrm{Si}$ III, N v) became weaker overall between Visit A and Visits D+E, while the flux in high-temperature lines $(\mathrm{O} v, \mathrm{Fe} \mathrm{XII})$ and X-rays increased. This could possibly trace a decrease in the chromospheric activity of HD 189733 from 2010 to 2013, while its corona became more active. The X-ray flux, in particular, increased significantly from visits B to the next visits. Over the same period the emission measures associated with each of

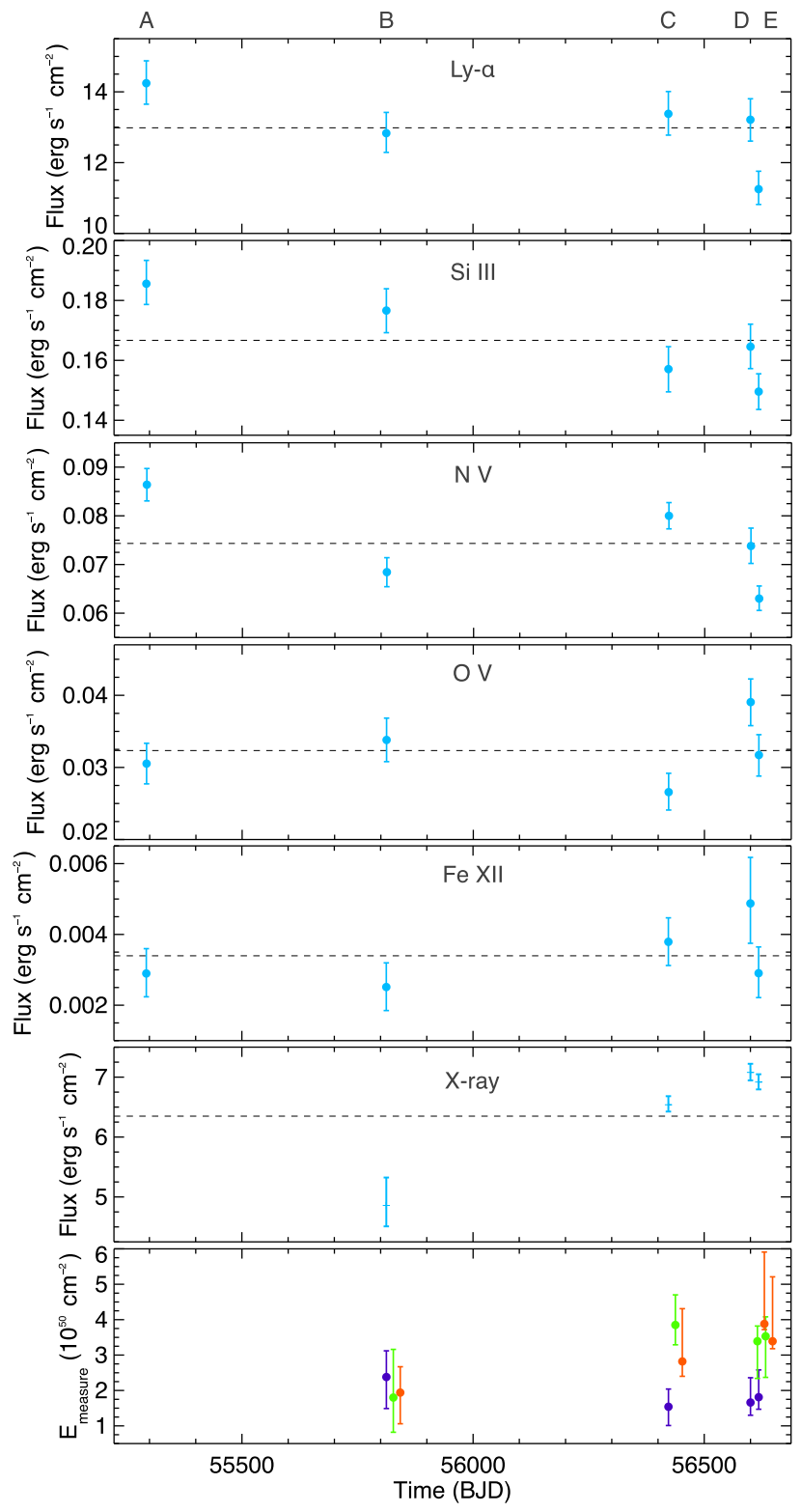

Figure 20. Emission from the chromosphere and corona of HD 189733 as a function of time. The upper panels show the total flux at 1 au from the star, in the theoretical intrinsic FUV lines fitted to the HST spectra (lines are ordered by increasing formation temperature from top to bottom) and in the $0.2-2.4 \mathrm{keV} X$-ray band (derived from the Swift and XMM-Newton spectra). The dashed black line is the mean flux over all epochs. The bottom panel shows the emission measures associated with the low (purple), medium (green), and high (orange) temperatures in the fitted X-ray spectra (slightly offset in each epoch for clarity).

the three X-ray temperature components remained consistent within their uncertainties, but the emission measures for the medium and high temperatures show a marginal increase. The second notable feature is the decrease in flux from Visit D to Visit E visible in all spectral bands, albeit stronger at low energies.

Both the increase in X-ray flux up to Visit D, and the global decrease in the emission of HD 189733 between visits D and E, correspond well to the evolution of the magnetic field over $9 \mathrm{yr}$ reported in Fares et al. (2017). In this study, we showed that the mean intensity of the magnetic field increased from $18 \mathrm{G}$ to $42 \mathrm{G}$ 
between 2006 and 2013 September (just before Visit D), before dropping sharply to $32 \mathrm{G}$ in 2014 September (after Visit E) and becoming more toroidal.

The intrinsic Lyman $\alpha$ line shows little variations in amplitude from Visit A to D, and keeps nearly the same shape (Fig. 16). Once rescaled to the same amplitude, the line profiles show nearly no variations in the wings and the peaks, and only slight variations in the depth of the self-reversal. In Visit E however, the line not only becomes weaker but also shows a shallower self-reversed core, which likely traces changes in the structure of the transition region associated with the aforementioned variations in the structure of the magnetic field and corona.

\section{INTERPRETATION OF THE FUV ABSORPTION SIGNATURES}

Our goal in this section is to qualitatively explore scenarios that could explain the observed FUV variations. Detailed modelling of HD 189733b upper atmosphere will be carried out in following papers of the MOVES series.

\subsection{Si III and N v lines}

Flux decreases were observed in the Si III line before and/or during the transit in Visits A and B. In Visits B and E the weaker line of the $\mathrm{N}$ V doublet showed variability, even though the brighter doublet line remained stable.

We used the synthetic stellar spectra derived in Section 4.4 to calculate photoionization lifetimes at the orbital distance of HD 189733 b. On average it takes $\sim 35 \mathrm{~h}$ for a neutral nitrogen atom to be ionized into $\mathrm{N}^{4+}$ (lifetimes associated with successive ionizations are $7.4+19.9+86.2+1989.2 \mathrm{~min}$ ). It would thus be unlikely for nitrogen atoms escaping the planet to remain in its vicinity long enough to be photoionized four times, which is in agreement with the non-detection of absorption in the brightest line of the $\mathrm{NV}$ doublet. The variations observed both in emission and absorption in the weaker line of the doublet (Sections 3.2 and 3.5), if they are not artefacts due to the lower SNR, likely trace stellar activity leading to density variations in the dominant emission regions of the corona.

In contrast, it takes $\sim 2 \mathrm{~h}$ for a neutral silicon atom to be ionized into $\mathrm{Si}^{2+}$ (successive lifetimes are $0.5+117.5 \mathrm{~min}$ ). Ionized silicon atoms escaped from the planet could thus remain in its vicinity and absorb the flux in the stellar Si III line near the transit, before they are carried away by stellar wind, radiation pressure, or magnetic interactions. However the escape of planetary silicon atoms would not explain why they were only detected in Visits A and B, and why they yield absorption only before/during the planetary transit in those visits. The change in the stellar spectral energy distribution (SED) from Visit $\mathrm{B}$ to $\mathrm{E}$, which results in respective photoionization lifetimes of 2.3, 1.7, 1.9, and $2.0 \mathrm{~h}$, does not explain these differences. We also note that the non-detection of Lyman $\alpha$ absorption in the same time windows as the Si III signatures in Visits $\mathrm{A}$ and $\mathrm{B}$ shows that the absorber is in a high ionization state. Another scenario proposed by Bourrier et al. (2013) is that the encounter of the stellar wind with the planet magnetosphere leads to the formation of a shock in which metals like silicon could quickly get ionized in a dense front ahead of the planet. The orientation and stand-off distance of this bow-shock would vary over time depending on the velocity of the stellar wind and the strength of the planet magnetic field (e.g. Vidotto et al. 2010; Llama et al. 2011). In this scenario, absorption of the Si III line just before and during the transit in Visit

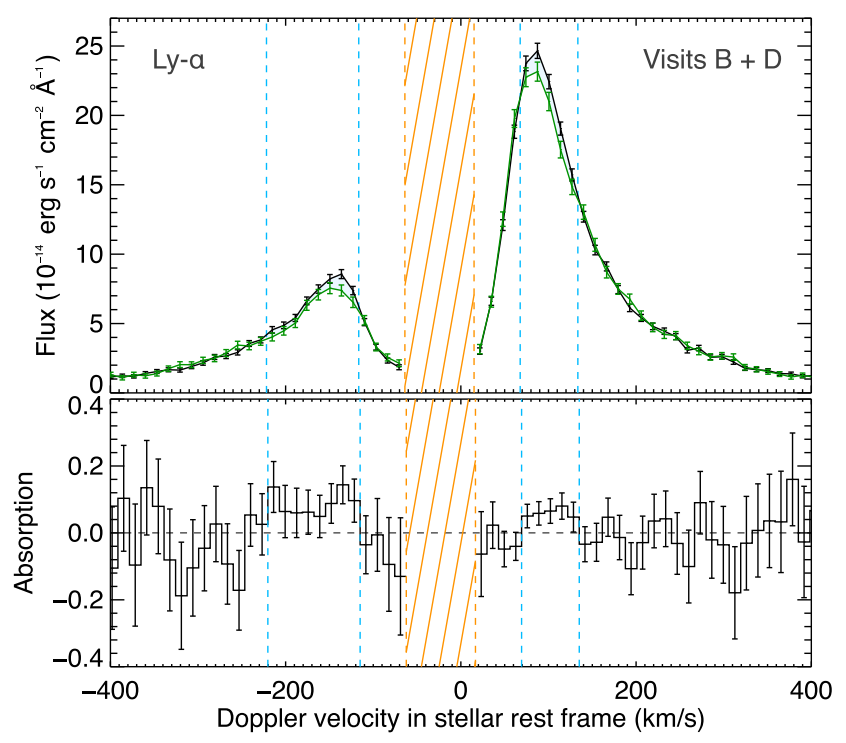

Figure 21. Top panel: Stellar Lyman $\alpha$ line averaged over visits B and D. Spectral ranges showing absorption during the transit (green spectrum) compared to pre-transit orbits (black spectrum) are highlighted in blue. The dashed orange region is contaminated by the airglow in Visit D. Bottom panel: Absorption spectrum defined as the relative flux ratio between the inand pre-transit spectra.

A would imply that the shock is facing the star more than in Visit $\mathrm{B}$, when it is only visible before the transit. This could result from a faster stellar wind in Visit A (see the extreme cases of daysideand ahead-shocks in Vidotto et al. 2010). The non-detection of the shock in subsequent visits could be linked to inhomogeneities in the density of the stellar wind along the planetary orbit (Llama et al. 2013; Kavanagh et al. 2019), resulting in a shock not dense enough to absorb the stellar lines. Alternatively it could be linked with the significant increase in X-ray emission, which traces an evolution of the coronal and stellar wind properties. For example higher coronal and wind temperatures could result in a more energetic shock that would ionize silicon atoms to higher levels than $\mathrm{Si}^{2+}$. We note that silicon atoms escaping from HD $189733 \mathrm{~b}$ are not required in this scenario, as the ionized population within the shock could originate from the stellar wind.

\subsection{Lyman $\alpha$ line}

In the following subsections we show how the absorption signatures observed in the Lyman $\alpha$ line in Visits B, D, and E could arise from the upper atmosphere of HD 189733b, and how their variable properties and the non-detection in Visit A could be linked to the evolution of the stellar SED and stellar wind.

\subsubsection{Visits $B$ and $D$}

As mentioned in Section 3.4 these two visits show the most similarities. To highlight this point we average their pre- and intransit Lyman $\alpha$ line spectra in Fig. 21. The signature at the peak of the red wing is more clearly revealed, although the absorption depth in excess of the planetary continuum remains marginal $\left(3.6 \pm 1.7\right.$ percent between 68 and $\left.134 \mathrm{~km} \mathrm{~s}^{-1}\right)$. The absorption signature in the blue wing, which extends over larger velocities in 
Visit B, yields an average excess absorption of $6.4 \pm 2.3$ percent between -222 and $-117 \mathrm{~km} \mathrm{~s}^{-1}$.

If confirmed, absorption in the red wing could possibly arise from the extended thermosphere of HD 189733b (Ben-Jaffel 2008; Guo \& Ben-Jaffel 2016). The similar levels of EUV irradiation and hydrogen photoionization rates in Visits B and D (Table 3) would lead to similar structures for the layer of neutral hydrogen, explaining the repeatability of the absorption signature. In contrast to Guo \& Ben-Jaffel (2016), however, we do not believe that the red wing absorption can arise entirely from the Lorentzian wings of the thermospheric absorption profile (the so-called damping wings). In this case, absorption should increase towards low velocities in the planet rest frame (see e.g. Bourrier et al. 2018b), whereas no absorption is detected in Visit B or D between $\sim 30$ and $70 \mathrm{~km} \mathrm{~s}^{-1}$ (Fig. 16).

Because of ISM absorption, blueshifted velocities lower than about $-100 \mathrm{~km} \mathrm{~s}^{-1}$ cannot be probed, preventing us from searching for the symmetrical signature expected from an extended thermosphere. The absorption signatures measured in the blue wing, at much larger velocities than in the red wing, cannot be due to the extended thermosphere. They also cannot be explained by radiation pressure, which requires more than $7 \mathrm{~h}$ to accelerate neutral hydrogen atoms escaping the planet to a terminal radial velocity of $\sim 140 \mathrm{~km} \mathrm{~s}^{-1}$ (Bourrier \& Lecavelier des Etangs 2013). The observed signatures could be explained by charge-exchange between the stellar wind and the planetary exosphere, which would create a population of energetic neutral hydrogen atoms (i.e. former stellar wind protons that got neutralized) moving with the velocity distribution of the stellar wind (Lecavelier des Etangs et al. 2012; Bourrier \& Lecavelier des Etangs 2013). In this scenario, the different velocity ranges measured in Visits B $\left(-220.3\right.$ to $\left.-128.1 \mathrm{~km} \mathrm{~s}^{-1}\right)$ and Visit $\mathrm{D}\left(-156.1\right.$ and $\left.-116.5 \mathrm{~km} \mathrm{~s}^{-1}\right)$ could trace variations in the stellar wind properties. As shown in Bourrier \& Lecavelier des Etangs (2013), the neutralized stellar wind protons are expected to trail the planet over a short distance before they are photoionized (lifetime $\sim 50 \mathrm{~min}$ ), which would further explain the absence of absorption after the transit in Visit B and at egress in Visit D (Sections 3.2 and 3.4). We thus favour interactions between the wind of HD 189733 and the exosphere of HD 189733b as the source for the measured Lyman $\alpha$ blueshifted absorption signatures.

\subsubsection{Visit A}

Lecavelier des Etangs et al. (2012), Bourrier \& Lecavelier des Etangs (2013) proposed that the atmospheric escape detected in Visit B was linked to the increased energy input and/or different stellar wind properties associated with the flare that occurred about $8 \mathrm{~h}$ before the transit. However, Chadney et al. (2017) showed that the short duration and energy spectrum typical of a flare on HD 189733, while increasing the total atmospheric loss from the planet, enhances neutral hydrogen loss by at most a factor of 2 . This is not sufficient to explain the increase in absorption depth from Visit A to Visit B (Bourrier \& Lecavelier des Etangs 2013). Furthermore if the observed blueshifted absorption signatures arise from stellar wind protons associated with a flare, the neutralized proton tail would have had to form and remain neutral within a limited time window after the flare, suggesting the stellar wind would only be enhanced for a short duration. It seems unlikely that we would have observed the transit of a tail in both Visits B, $\mathrm{D}$, and $\mathrm{E}$ (see below) at the right time a few hours after a stellar flare.
Another possibility to explain the difference between Visit A and subsequent visits is the change in the stellar SED. If the Xray emission followed the same trend as in other visits, it was significantly lower in Visit A (Fig. 20), likely decreasing the global extension of the thermosphere and its mass-loss rate. Meanwhile Visit A shows the largest flux of all visits in the Lyman $\alpha$ and Si III lines, implying that the EUV irradiation on the planet was highest in this epoch and that the hydrogen layer within the thermosphere was more ionized. Guo \& Ben-Jaffel (2016) suggested that an increase in the ratio $F(50-400 \AA) / F(50-900 \AA)$ by a factor 2 from Visit A to Visit $B$ would have made the thermosphere dense enough in neutral hydrogen for its damping wings to become detectable in the red wing of the Lyman $\alpha$ line in Visit B. However we note that a change in the thermospheric structure does not explain by itself the variations observed in the blue wing of the line, where absorption signatures are measured at velocities too high to arise from the thermosphere. It is the reduced escape rate of neutral hydrogen from the thermosphere, combined with the higher photoionization of escaping hydrogen atoms, which could have limited the abundance of neutral hydrogen atoms arising from charge exchange with the exosphere in Visit A. For example Bourrier \& Lecavelier des Etangs (2013) showed that photoionization rates at least three times larger in Visit A than in Visit B, with similar escape rates of neutral hydrogen, would have prevented the formation of a detectable neutralized proton tail detectable in the blue wing of the Lyman $\alpha$ line.

\subsubsection{Visit E}

There are strong similarities between Visit E and Visits B+D. In the two cases, absorption signatures are measured during the transit, in both wings of the Lyman $\alpha$ line and from about the same velocities (70 $\mathrm{km} \mathrm{s}^{-1}$ in the red wing, $-110 \mathrm{~km} \mathrm{~s}^{-1}$ in the blue wing), while the core of the line remains stable. On the other hand, signatures are deeper in both wings of the line in Visit E, and extend up to larger velocities than in Visits $B+D$.

Interestingly, Visit E shows the lowest flux of all visits in lowenergy chromospheric lines and one of the largest X-ray emission, in particular at soft energies mostly responsible for heating in the upper atmosphere (Fig. 20, Table 3). This evolution in the stellar SED could have led to a dramatic change in the upper atmosphere of HD 189733b. Based on the calculations by Owen \& Jackson (2012), HD189733b is close to the limit where the escape regime transitions from being EUV- to X-ray driven (see their fig. 11 , with $\mathrm{a}=0.032 \mathrm{au}, \rho=0.8 \mathrm{~g} \mathrm{~cm}^{-3}, \mathrm{~L}_{\mathrm{x}}(\lambda<100 \AA)$ $\sim 3 \times 10^{28} \mathrm{erg} \mathrm{s}^{-1}$ in Visit E). This transition might have occurred in Visit E, leading to larger mass-loss in the X-ray-driven regime (Owen \& Jackson 2012). The low photoionization rates in this epoch would have further increased the abundance of neutral hydrogen in the escaping outflow, amplifying absorption signatures in this epoch. The similarities of the absorption signatures in Visit B, D, and $E$ at low velocities suggest that the dynamics of the neutral hydrogen layer close to the planet remains controlled by the same mechanism. The larger velocities of the red wing signature in Visit E suggest that we probe neutral hydrogen gas moving farther from the planet, possibly a stream accreting towards the star revealed by the enhancement in neutral hydrogen abundance (Lai, Helling \& van den Heuvel 2010; Lanza 2014; Matsakos et al. 2015; Strugarek 2016). Such a stream could yield transit signatures in the Balmer lines at even larger distance from the planet, as suggested by the detection of pre- and post-transit absorption in ground-based optical 
observations (see Cauley et al. 2017 and references within). The larger velocities of the blue wing signature in Visit E might trace larger local velocities of the stellar wind at the orbit of the planet. Residual absorption might have been detected in additional posttransit observations from a tail trailing the planet, in contrast to Visit B.

\section{DISCUSSIONS}

The corona of HD 189733 became more active over the course of our observations while chromospheric activity decreased, which likely led to substantial changes in the planetary environment. In particular the variations observed in X-ray emission correlate well with the evolution of the stellar magnetosphere described in Fares et al. (2017). The stellar wind properties in Visits A and B, when the stellar magnetic and coronal activity was reduced, could have favoured the formation of a dense population of $\mathrm{Si}^{2+}$ atoms in a bow-shock ahead of the planet, responsible for the pre- and in-transit absorption measured in the Si III line in those visits. Meanwhile, we surmise that a lower X-ray irradiation and larger photoionization of the planet in Visit A could have limited the extension and neutral content of its upper atmosphere, explaining why no Lyman $\alpha$ transit was detected in this epoch. In subsequent visits, the change in stellar SED may have increased the abundance of neutral hydrogen in the thermosphere, which could be partly responsible for the absorption signatures detected in Visits B, D, and $\mathrm{E}$ at low velocities in the red wing of the Lyman $\alpha$ line. The corresponding increase in neutral hydrogen escape would further explain the absorption signatures observed in those visits at high velocities in the blue wing of the line, arising from charge-exchange between the variable stellar wind and the (neutral) hydrogen exosphere. A sharp change in the structure of the star magnetosphere and its high-energy emission from Visit D to Visit E might then have led to a dramatic change in the evaporation regime of the planet, sustaining a much larger neutral hydrogen loss. In any individual epoch, no transit signatures were detected in the $\mathrm{NV}$ and $\mathrm{OV}$ lines, or in the X-rays.

Based on these results, we make the following predictions:

(i) epochs of low EUV emission and high X-ray emission enhance the abundance of neutral hydrogen in the thermosphere and the exosphere of HD 189733b, and are thus the most favourable to probe the upper atmosphere via Lyman $\alpha$ transit spectroscopy.

(ii) absorption from the upper atmosphere is maximal during the time window of the planetary transit.

(iii) epochs of low X-ray emission, possibly associated with a less energetic stellar wind, lead to the formation of a bow-shock enriched in low-ionization species and with a different orientation.

Future observations of the planetary environment could thus be planned based on the predicted stellar activity level, and should monitor the stellar X-ray emission while searching for the transit of the planet upper atmosphere and escaping outflow in FUV lines and other potential tracers.

Overall, the XUV irradiation of HD $189733 \mathrm{~b}$ is high enough in all epochs for the upper atmosphere to be extended and escaping (e.g. Owen \& Jackson 2012). The detection of repeatable absorption signatures in three independent epochs from an extended but compact helium layer (Salz et al. 2018) further suggests that the lower regions of the extended thermosphere are stable over time. However, our study shows how variations in the stellar XUV spectrum and wind properties could influence the density of neutral hydrogen in the upper thermosphere and in the exosphere, resulting in the variability of their observed Lyman $\alpha$ transit signatures. It is worth noting that the Lyman $\alpha$ blueshifted absorption signatures would trace the local conditions of the stellar wind. Observations of the solar wind (e.g. McComas et al. 2008) and numerical simulations of stellar winds (Llama et al. 2013; Kavanagh et al. 2019) have shown that winds are not homogeneous, with streams of highand low-velocity material coexisting at any given epoch. These inhomogeneities are linked to the topology of stellar magnetic fields, which for HD 189733b is known to be complex and evolve over time (Fares et al. 2017). This variability of HD 189733b upper atmosphere was hinted by previous unresolved observations of the Lyman $\alpha$ line with HST/ACS (Lecavelier des Etangs et al. 2010), which showed consistent excess atmospheric absorption depths of $5.2 \pm 1.5$ per cent on 2007 June 10 and $3.2 \pm 1.7$ per cent on 2007 June 18/19 (four transits later), but no excess in a third visit in 2008 April. We note, however, that a flare observed during the 2008 April visit might have contaminated the observations. Our study revealed another flare from the primary star that occurred during the transit in Visit C, with a FUV-signature only. Flares occurring repeatedly after the secondary eclipse have been proposed as a signature of SPI between HD 189733b and its star (Pillitteri et al. $2010,2011,2014)$. In contrast, despite many transit observations of HD 189733 b this is only the third time that a flare is observed during the primary transit (the first was measured in the FUV by Lecavelier des Etangs et al. 2010, the second in optical chromospheric lines by Klocová et al. 2017), making it unlikely that they are induced by the planet.

\section{CONCLUSIONS}

This paper is part of the MOVES collaboration, which aims at characterizing the environment of the hot Jupiter HD 189733b and its star via multiwavelength observations obtained contemporaneously in different epochs. In MOVES I (Fares et al. 2017) we used optical spectropolarimetry to reconstruct the 3D stellar magnetosphere and study its evolution over several years. These results were used in MOVES II (Kavanagh et al. 2019) to model the wind of the host star and study its influence on the planetary radio emission. In this paper (MOVES III), we combined FUV and X-ray observations to characterize the stellar high-energy emission, to search for transit signatures from the planet upper atmosphere, and to study their evolution in five epochs from 2010 to 2013.

Transit signatures are measured in the stellar Lyman $\alpha$ line in three epochs, and in the Si III line in two epochs. These signatures could be related to the evolution of the stellar high-energy emission and stellar wind (MOVES II), linked to the evolution of the stellar magnetosphere (MOVES I). Our analysis thus confirms the evaporation of HD $189733 \mathrm{~b}$ and its temporal variability, and supports the presence of a bow-shock ahead of the planet.

Knowledge of the stellar irradiation is key to our understanding of close-in planet atmospheres. Their photochemistry and stability is influenced by XUV photons, making critical the use of realistic stellar spectra in atmospheric models. Previous studies of HD 189733 b have used a variety of approaches, including the solar EUV spectrum, stellar proxys like $\epsilon$ Eridiani, or theoretical spectra (see discussion in Guo \& Ben-Jaffel 2016). Here we combined measurements of the soft X-ray emission and chromospheric/transition region lines from HD 189733 to constrain a model of the stellar atmosphere and reconstruct its entire XUV spectrum in four epochs. These synthetic spectra, which extend up to $1600 \AA$, are available online. The Lyman $\alpha$ line of HD 189733 alone represents half of the flux emitted in the entire EUV domain. It is particularly important 
for atmospheric chemistry (e.g. Miguel et al. 2015) but also for the structure of the planetary exosphere affected by radiation pressure. We thus include our reconstructed profiles for the intrinsic Lyman $\alpha$ line of HD 189733 in its synthetic spectra. This reconstruction further allowed us to characterize the ISM properties towards HD 189733, revealing at least two clouds along the LOS.

Future studies of the MOVES collaboration will combine the stellar wind models from MOVES II with the synthetic stellar XUV spectra derived here to inform detailed models of HD 189733b upper atmosphere, and determine the mechanisms and physical properties responsible for the observed FUV signatures.

\section{ACKNOWLEDGEMENTS}

We thank the referee for their attentive review of our paper. We thank D.K. Sing for his help and insights into the investigation of the HST jitter. VB acknowledges support by the Swiss National Science Foundation (SNSF) in the frame of the National Centre for Competence in Research 'PlanetS', and has received funding from the European Research Council (ERC) under the European Union's Horizon 2020 research and innovation programme (project Four Aces; grant agreement No. 724427). PJW, GK, and TL acknowledge support from the UK Science and Technology Facilities Council (STFC) under the consolidated grants ST/L000733/1 and ST/P000495/1. ALE acknowledges support from the French Centre national d'Etude Spatiales (CNES) and the French Agence Nationale de la Recherche (ANR), under programme ANR-12BS05-0012 Exo-Atmos. AAV acknowledges funding from the Irish Research Council. This work is based on observations made with the National Aeronautics and Space Administration / European Space Agency (NASA/ESA) Hubble Space Telescope, obtained at the Space Telescope Science Institute, which is operated by the Association of Universities for Research in Astronomy, Inc., under NASA contract NAS 5-26555. This work is based on observations obtained with XMM-Newton, an ESA science mission with instruments and contributions directly funded by ESA Member States and NASA. This research has made use of data obtained from NASA's Swift satellite. This work has made use of data from the ESA mission Gaia (https://www.cosmos.esa.int/gaia), processed by the Gaia Data Processing and Analysis Consortium (DPAC, ht tps://www.cosmos.esa.int/web/gaia/dpac/consortium). Funding for the DPAC has been provided by national institutions, in particular the institutions participating in the Gaia Multilateral Agreement.

\section{REFERENCES}

Achour H., Brekke P., Kjeldseth-Moe O., Maltby P., 1995, ApJ, 453, 945

Agol E., Cowan N. B., Knutson H. A., Deming D., Steffen J. H., Henry G. W., Charbonneau D., 2010, ApJ, 721, 1861

Arnaud K. A., 1996, in Jacoby G. H., Barnes J., eds, ASP Conf. Ser. Vol. 101, Astronomical Data Analysis Software and Systems V. Astron. Soc. Pac., San Francisco, p. 17

Aurière M., 2003, in Arnaud J., Meunier N., eds, EAS Publications Series Vol. 9, EAS Publications Series. EAS Publications, Nairobi, Kenya, p. 105

Ayres T. R., 2015, AJ, 150, 7

Bakos G. Á. et al., 2006, ApJ, 650, 1160

Ballester G. E., Ben-Jaffel L., 2015, ApJ, 804, 116

Baluev R. V., 2015, MNRAS, 446, 1493

Beaugé C., Nesvorný D., 2013, ApJ, 763, 12

Ben-Jaffel L., 2008, ApJ, 688, 1352

Ben-Jaffel L., Ballester G. E., 2013, A\&A, 553, A52
Ben-Jaffel L., Sona Hosseini S., 2010, ApJ, 709, 1284

Bouchy F. et al., 2005, A\&A, 444, L15

Bourrier V., Lecavelier des Etangs A., 2013, A\&A, 557, A124

Bourrier V. et al., 2013, A\&A, 551, A63

Bourrier V., Ehrenreich D., Lecavelier des Etangs A., 2015, A\&A, 582, A65

Bourrier V., Lecavelier des Etangs A., Ehrenreich D., Tanaka Y. A., Vidotto A. A., 2016, A\&A, 591, A121

Bourrier V., Ehrenreich D., King G., Lecavelier des Etangs A., Wheatley P. J., Vidal-Madjar A., Pepe F., Udry S., 2017, A\&A, 597, A26

Bourrier V. et al., 2018a, A\&A, 615, A117

Bourrier V. et al., 2018b, A\&A, 620, A147

Brown T. M., Charbonneau D., Gilliland R. L., Noyes R. W., Burrows A., 2001, ApJ, 552, 699

Burrows D. N. et al., 2005, Space Sci. Rev., 120, 165

Caffau E., Ludwig H.-G., Steffen M., Freytag B., Bonifacio P., 2011, Solar Phys., 268, 255

Cauley P. W., Redfield S., Jensen A. G., Barman T., Endl M., Cochran W. D., 2015, ApJ, 810, 13

Cauley P. W., Redfield S., Jensen A. G., Barman T., 2016, AJ, 152, 20

Cauley P. W., Redfield S., Jensen A. G., 2017, AJ, 153, 217

Cauley P. W., Shkolnik E. L., Llama J., Bourrier V., Moutou C., 2018, AJ, 156,262

Chadney J. M., Koskinen T. T., Galand M., Unruh Y. C., Sanz-Forcada J., 2017, A\&A, 608, A75

Cohen O., Kashyap V. L., Drake J. J., Sokolov I. V., Garraffo C., Gombosi T. I., 2011, ApJ, 733, 67

Cuntz M., Saar S. H., Musielak Z. E., 2000, ApJ, 533, L151

Czesla S., Klocová T., Khalafinejad S., Wolter U., Schmitt J. H. M. M., 2015, A\&A, 582, A51

Davis T. A., Wheatley P. J., 2009, MNRAS, 396, 1012

Dere K. P., Landi E., Mason H. E., Monsignori Fossi B. C., Young P. R., 1997, A\&AS, 125

Donati J. F., 2003, in Trujillo-Bueno J., Almeida J. S., eds, ASP Conf. Ser. Vol. 307, ESPaDOnS: An Echelle SpectroPolarimetric Device for the Observation of Stars at CFHT. Astron. Soc. Pac., San Francisco, p. 41

Donati J. F., Catala C., Landstreet J. D., Petit P., 2006, in Casini R., Lites B. W., eds, ASP Conf. Ser. Vol. 358, ESPaDOnS: The New Generation Stellar Spectro-Polarimeter. Performances and First Results. Astron. Soc. Pac., San Francisco, p. 362

Eastman J., Gaudi B. S., Agol E., 2013, PASP, 125, 83

Ehrenreich D. et al., 2008, A\&A, 483, 933

Ehrenreich D., Lecavelier des Etangs A., Delfosse X., 2011, A\&A, 529, A80

Ehrenreich D. et al., 2012, A\&A, 547, A18

Ehrenreich D. et al., 2015, Nature, 522, 459

Fares R. et al., 2010, MNRAS, 406, 409

Fares R. et al., 2017, MNRAS, 471, 1246

Feldman U., 1992, Phys. Scr, 46, 202

Foreman-Mackey D., Hogg D. W., Lang D., Goodman J., 2013, PASP, 125, 306

Fossati L. et al., 2010, ApJ, 714, L222

Gaia Collaboration, 2018, A\&A, 616, A1

García Muñoz A., 2007, Planet. Space Sci., 55, 1426

Guo J. H., Ben-Jaffel L., 2016, ApJ, 818, 107

Hannah I. G., Kontar E. P., 2012, A\&A, 539, A146

Hansteen V. H., Hara H., De Pontieu B., Carlsson M., 2010, ApJ, 718, 1070

Haswell C. A. et al., 2012, ApJ, 760, 79

Hébrard G., Moos H. W., 2003, ApJ, 599, 297

Hodge P. E. et al., 1998, in Albrecht R., Hook R. N., Bushouse H. A., eds, ASP Conf. Ser. Vol. 145, Pipeline Calibration for STIS. Astron. Soc. Pac., San Francisco, p. 316

Hubbard W. B., Hattori M. F., Burrows A., Hubeny I., Sudarsky D., 2007, Icarus, 187,358

Huitson C. M., Sing D. K., Vidal-Madjar A., Ballester G. E., Lecavelier des Etangs A., Désert J.-M., Pont F., 2012, MNRAS, 422, 2477

Ip W.-H., Kopp A., Hu J.-H., 2004, ApJ, 602, L53

Jensen A. G., Redfield S., Endl M., Cochran W. D., Koesterke L., Barman T., 2012, ApJ, 751, 86 
Jin S., Mordasini C., Parmentier V., van Boekel R., Henning T., Ji J., 2014, ApJ, 795, 65

Johnstone C. P. et al., 2015, ApJ, 815, L12

Kavanagh R. D. et al., 2019, MNRAS, 485, 4529

Klocová T., Czesla S., Khalafinejad S., Wolter U., Schmitt J. H. M. M., 2017, A\&A, 607, A66

Kohl S., Salz M., Czesla S., Schmitt J. H. M. M., 2018, A\&A, 619, A96

Koskinen T. T., Yelle R. V., Lavvas P., Lewis N. K., 2010, ApJ, 723, 116

Kramida A., Ralchenko Y., Reader J., 2016, APS Division of Atomic, Molecular and Optical Physics Meeting Abstracts. Am. Phys. Soc., Providence, RI

Kulow J. R., France K., Linsky J., Loyd R. O. P., 2014, ApJ, 786, 132

Kurokawa H., Nakamoto T., 2014, ApJ, 783, 54

Lai D., Helling Ch., van den Heuvel E. P. J., 2010, ApJ, 721, 923

Laming J. M., 2015, Living Rev. Solar Phys., 12, 2

Lammer H., Selsis F., Ribas I., Guinan E. F., Bauer S. J., Weiss W. W., 2003, ApJ, 598, L121

Landi E., Del Zanna G., Young P. R., Dere K. P., Mason H. E., 2012, ApJ, 744, 99

Lanza A. F., 2014, A\&A, 572, L6

Lavie B. et al., 2017, A\&A, 605, L7

Lecavelier des Etangs A., 2007, A\&A, 461, 1185

Lecavelier des Etangs A., Vidal-Madjar A., McConnell J. C., Hébrard G., 2004, A\&A, 418, L1

Lecavelier des Etangs A. et al., 2010, A\&A, 514, A72

Lecavelier des Etangs A. et al., 2012, A\&A, 543, L4

Liddle A. R., 2007, MNRAS, 377, L74

Linsky J. L. et al., 2006, ApJ, 647, 1106

Linsky J. L., Yang H., France K., Froning C. S., Green J. C., Stocke J. T., Osterman S. N., 2010, ApJ, 717, 1291

Linsky J. L., Bushinsky R., Ayres T., France K., 2012, ApJ, 754, 69

Llama J., Shkolnik E. L., 2015, ApJ, 802, 41

Llama J., Wood K., Jardine M., Vidotto A. A., Helling C., Fossati L., Haswell C. A., 2011, MNRAS, 416, L41

Llama J., Vidotto A. A., Jardine M., Wood K., Fares R., Gombosi T. I., 2013, MNRAS, 436, 2179

Lopez E. D., Fortney J. J., 2013, ApJ, 776, 2

Lopez E. D., Fortney J. J., Miller N., 2012, ApJ, 761, 59

Louden T., Wheatley P. J., Briggs K., 2017, MNRAS, 464, 2396

Loyd R. O. P. et al., 2018, ApJ, 867, 71

Lundkvist M. S. et al., 2016, Nat. Commun., 7, 11201

Mandel K., Agol E., 2002, ApJ, 580, L171

Matsakos T., Uribe A., Königl A., 2015, A\&A, 578, A6

McComas D. J., Ebert R. W., Elliott H. A., Goldstein B. E., Gosling J. T., Schwadron N. A., Skoug R. M., 2008, Geophys. Res. Lett., 35, L18103

Miguel Y., Kaltenegger L., Linsky J. L., Rugheimer S., 2015, MNRAS, 446, 345

Mitra-Kraev U. et al., 2005, A\&A, 431, 679

Moutou C. et al., 2007, A\&A, 473, 651

Owen J. E., Jackson A. P., 2012, MNRAS, 425, 2931

Owen J. E., Wu Y., 2013, ApJ, 775, 105

Peter H., Judge P. G., 1999, ApJ, 522, 1148

Pillitteri I., Wolk S. J., Cohen O., Kashyap V., Knutson H., Lisse C. M., Henry G. W., 2010, ApJ, 722, 1216

Pillitteri I., Günther H. M., Wolk S. J., Kashyap V. L., Cohen O., 2011, ApJ, 741, L18

Pillitteri I., Wolk S. J., Lopez-Santiago J., Günther H. M., Sciortino S., Cohen O., Kashyap V., Drake J. J., 2014, ApJ, 785, 145
Pillitteri I., Maggio A., Micela G., Sciortino S., Wolk S. J., Matsakos T., 2015, ApJ, 805, 52

Pino L. et al., 2018, A\&A, 612, A53

Poppenhaeger K., Schmitt J. H. M. M., 2011, Astron. Nachr., 332, 1052

Poppenhaeger K., Schmitt J. H. M. M., Wolk S. J., 2013, ApJ, 773, 62

Redfield S., Linsky J. L., 2008, ApJ, 673, 283

Route M., 2019, ApJ, 872, 79

Salz M. et al., 2018, A\&A, 620, A97

Scandariato G. et al., 2013, A\&A, 552, A7

Schlawin E., Agol E., Walkowicz L. M., Covey K., Lloyd J. P., 2010, ApJ, 722, L75

Schmelz J. T., Reames D. V., von Steiger R., Basu S., 2012, Astrophys. J., 755,33

Schneiter E. M., Esquivel A., D’Angelo C. S. V., Velázquez P. F., Raga A. C., Costa A., 2016, MNRAS, 457, 1666

Shkolnik E., Walker G. A. H., Bohlender D. A., 2003, ApJ, 597, 1092

Shkolnik E., Bohlender D. A., Walker G. A. H., Collier Cameron A., 2008, ApJ, 676, 628

Sing D. K., Vidal-Madjar A., Désert J.-M., Lecavelier des Etangs A., Ballester G., 2008, ApJ, 686, 658

Smith R. K., Brickhouse N. S., Liedahl D. A., Raymond J. C., 2001, ApJ, 556, L91

Strüder L. et al., 2001, A\&A, 365, L18

Strugarek A., 2016, ApJ, 833, 140

Szabó G. M., Kiss L. L., 2011, ApJ, 727, L44

Tremblin P., Chiang E., 2013, MNRAS, 428, 2565

Triaud A. H. M. J. et al., 2009, A\&A, 506, 377

van Haarlem M. P. et al., 2013, A\&A, 556, A2

Vidal-Madjar A., Lecavelier des Etangs A., Désert J.-M., Ballester G. E., Ferlet R., Hébrard G., Mayor M., 2003, Nature, 422, 143

Vidal-Madjar A. et al., 2004, ApJ, 604, L69

Vidal-Madjar A., Lecavelier des Etangs A., Désert J.-M., Ballester G. E., Ferlet R., Hébrard G., Mayor M., 2008, ApJ, 676, L57

Vidal-Madjar A. et al., 2013, A\&A, 560, A54

Vidotto A. A., Jardine M., Helling C., 2010, ApJ, 722, L168

Wilms J., Allen A., McCray R., 2000, ApJ, 542, 914

Wood B. E., Linsky J. L., Hébrard G., Williger G. M., Moos H. W., Blair W. P., 2004, ApJ, 609, 838

Wood B. E., Redfield S., Linsky J. L., Müller H.-R., Zank G. P., 2005, ApJS, 159,118

Wood B. E., Laming J. M., Karovska M., 2012, ApJ, 753, 76

Woodgate B. E. et al., 1998, PASP, 110, 1183

Yelle R. V., 2004, Icarus, 170, 167

Youngblood A. et al., 2016, ApJ, 824, 101

Youngblood A. et al., 2017, ApJ, 843, 31

\section{SUPPORTING INFORMATION}

Supplementary data are available at MNRAS online.

Please note: Oxford University Press is not responsible for the content or functionality of any supporting materials supplied by the authors. Any queries (other than missing material) should be directed to the corresponding author for the article.

This paper has been typeset from a $\mathrm{T}_{\mathrm{E}} \mathrm{X} / \mathrm{L} \mathrm{T} \mathrm{E} \mathrm{X}$ file prepared by the author. 